\title{
MicroRNA-655-3p and microRNA-497-5p inhibit cell proliferation in cultured human lip cells through the regulation of genes related to human cleft lip
}

Mona Gajera ${ }^{1 \dagger}$, Neha Desai ${ }^{1 \dagger}$, Akiko Suzuki ${ }^{1,2}$, Aimin $\mathrm{Li}^{3}$, Musi Zhang ${ }^{1,2}$, Goo Jun ${ }^{4,5}$, Peilin $\mathrm{Jia}^{3}$, Zhongming Zhao ${ }^{3,4,5}$ and Junichi Iwata ${ }^{1,2,5^{*}}$ (1)

\begin{abstract}
Background: The etiology of cleft lip with or without cleft palate (CL/P), a common congenital birth defect, is complex and involves the contribution of genetic and environmental factors. Although many candidate genes have been identified, the regulation and interaction of these genes in CL/P remain unclear. In addition, the contribution of microRNAs (miRNAs), non-coding RNAs that regulate the expression of multiple genes, to the etiology of $\mathrm{CL} / \mathrm{P}$ is largely unknown.

Methods: To identify the signatures of causative biological pathways for human CL/P, we conducted a systematic literature review for human CL/P candidate genes and subsequent bioinformatics analyses. Functional enrichment analyses of the candidate CL/P genes were conducted using the pathway databases GO and KEGG. The miRNA-mediated post-transcriptional regulation of the CL/P candidate genes was analyzed with miRanda, PITA, and TargetScan, and miRTarbase. Genotype-phenotype association analysis was conducted using GWAS. The functional significance of the candidate miRNAs was evaluated experimentally in cell proliferation and target gene regulation assays in human lip fibroblasts.

Results: Through an extensive search of the main biomedical databases, we mined 177 genes with mutations or association/linkage reported in individuals with CL/P, and considered them as candidate genes for human CL/P. The genotype-phenotype association study revealed that mutations in 12 genes (ABCA4, ADAM3A, FOXE1, IRF6, MSX2, MTHFR, NTN1, PAX7, TP63, TPM1, VAX1, and WNT9B) were significantly associated with CL/P. In addition, our bioinformatics analysis predicted 16 microRNAs (miRNAs) to be post-transcriptional regulators of CL/P genes. To validate the bioinformatics results, the top six candidate miRNAs (miR-124-3p, miR-369-3p, miR-374a-5p, miR-374b-5p, miR-497-5p, and miR-655-3p) were evaluated by cell proliferation/survival assays and miRNA-gene regulation assays in cultured human lip fibroblasts. We found that miR-497-5p and miR-655-3p significantly suppressed cell proliferation in these cells. Furthermore, the expression of the predicted miRNA-target genes was significantly downregulated by either miR-497-5p or miR-655-3p mimic.
\end{abstract}

(Continued on next page)

\footnotetext{
* Correspondence: Junichi.lwata@uth.tmc.edu

${ }^{\dagger}$ Mona Gajera and Neha Desai contributed equally to this work.

'Department of Diagnostic \& Biomedical Sciences, School of Dentistry, The

University of Texas Health Science Center at Houston, Houston, TX, USA

${ }^{2}$ Center for Craniofacial Research, The University of Texas Health Science

Center at Houston, Houston, TX, USA

Full list of author information is available at the end of the article
}

(c) The Author(s). 2019 Open Access This article is distributed under the terms of the Creative Commons Attribution 4.0 International License (http://creativecommons.org/licenses/by/4.0/), which permits unrestricted use, distribution, and reproduction in any medium, provided you give appropriate credit to the original author(s) and the source, provide a link to the Creative Commons license, and indicate if changes were made. The Creative Commons Public Domain Dedication waiver (http://creativecommons.org/publicdomain/zero/1.0/) applies to the data made available in this article, unless otherwise stated. 
(Continued from previous page)

Conclusion: Expression of miR-497-5p and miR-655-3p suppresses cell proliferation through the regulation of human CL/ P-candidate genes. This study provides insights into the role of miRNAs in the etiology of CL/P and suggests possible strategies for the diagnosis of CL/P.

Keywords: Cleft lip, Gene mutation, MicroRNA, Systematic review, Bioinformatics analysis

\section{Background}

Cleft lip with or without cleft palate $(\mathrm{CL} / \mathrm{P})$ is a common congenital malformation affecting speech, hearing, feeding, among others functions [1]. Individuals with $\mathrm{CL} / \mathrm{P}$ require a comprehensive treatment, including multiple plastic and maxillofacial surgeries from birth to adulthood, and speech therapy [2]. It has an average worldwide birth prevalence of 1 in 1000, ranging from 1 in 500 in the Asian population to 1 in 2500 in the African population, with wide variability per geographic origin, ethnicity, and socioeconomic status $[3,4]$. The complexity of its etiology seems to result from various genetic and environmental risk factors along with gene- environment interactions (e.g., approximately 70\% cases are non-syndromic) [5]. Genome-wide association studies (GWAS) and linkage studies have identified genetic susceptibility to CL/P and differences in such susceptibility in various populations and ethnic groups [6]. However, as seen in many complex diseases or traits, most of the mutations and loci identified from such studies are in the noncoding genomic regions and do not have specific functional annotations [7, 8]. Although much progress has been made in identifying genes whose mutations are associated with $\mathrm{CL} / \mathrm{P}$, little is known about the mechanisms by which environmental and epigenetic factors adversely influence gene expression during lip development. Recent studies indicate that environmental factors contribute to changes in phenotype or gene expression at post-transcriptional level through the regulation of noncoding RNAs, including microRNAs (miRNAs) [9]. miRNAs are short ( 22 nucleotides) noncoding RNA molecules that regulate gene expression at the post- transcriptional level, and they fine-tune the expression of $\sim 30 \%$ of all mammalian protein-encoding genes [10]. miRNAs were first reported in mammalian systems in 2001 [11], but the latest release of the miRNA database [miRBase (ver. 20): more than 24,000 miRs annotated] highlights the rapid growth of this field of research; however, the expression patterns and functions of most miRNAs still remain to be discovered. The miRNA-gene regulatory mechanisms contribute to the pathogenesis in various diseases $[12,13]$. Nonetheless, a limited number of miRNAs (e.g. miRNA140, miRNA-17-92 cluster, miRNA-200b, miRNA-133b) have been reported as miRNAs involved in craniofacial deformities in zebrafish and mouse models, as well as in humans [14]. Therefore, an investigation of the functional regulation, at the level of biological pathways and posttranscriptional regulation mechanism, will improve our understanding of genetic susceptibility to $\mathrm{CL} / \mathrm{P}[7,15]$.

In this study, in order to identify signatures of causative pathways in the complex etiology of CL/P, we performed a systematic review and subsequent bioinformatics analysis for $\mathrm{CL} / \mathrm{P}$-candidate genes. In addition, we analyzed miRNA-gene regulation by bioinformatics analyses. The function in cell proliferation/survival for six candidate miRNAs was experimentally evaluated by cell proliferation assays. Our findings will help us understand the role of genes and miRNAs that are associated with CL/P, in biological pathways and networks. Such knowledge will provide the basis for the diagnosis, prevention, and treatment of craniofacial anomalies such as CL/P.

\section{Methods}

\section{Data sources}

The publishing guidelines set forth by PRISMA (Preferred Reporting Items for Systematic Reviews and Meta- Analyses) were followed during the literature search and review. The search was conducted using three main literature databases: Medline (Ovid), PubMed (NLM), and Embase (Ovid). In addition, related citations were searched in Scopus (Elsevier, Inc.) to check whether any unique studies were missed from the regular database searches.

\section{Inclusion and exclusion criteria}

The articles meeting the following eligibility criteria were included in our systematic review:

- described genes causing or potentially associated with CL/P in humans;

- referred to studies of either syndromic or nonsyndromic CL/P;

- were published as original articles (not as review articles, editorials, dissertations, conference proceedings or comments);

- included cross-sectional, case-control, cohort studies, or clinical trials;

- were published in English.

After screening for articles using the criteria above, we manually excluded those articles in which: 
- gene mutations were not described in the original articles;

- $\mathrm{CL} / \mathrm{P}$ was not specifically described;

- $\mathrm{CL} / \mathrm{P}$ resulted from environmental factors;

- treatments for CL/P were described;

- were case reports;

- the articles failed to fit in any of the above criteria but did not have CL/P candidate genes or related information.

\section{Search methods to identify studies}

Concepts included in the search were: CL/P, genetics (gene mutations), and humans. A combination of Medical Subject Headings (MeSH) terms and titles, abstracts, and keywords was used to develop the initial Medline search string, and then adapted to search the other databases. The last search date was May 28, 2018.

\section{Study selection and data collection}

All the citations found in the search process were stored in RefWorks (ProQuest) and any duplicates were excluded. Search strategies and results were tracked using the Primary Excel Workbook designed for systematic reviews (http://libguides.sph.uth.tmc.edu/excel_SR_workbook). To check the reliability of study selection between the screeners, Cohen's Kappa test was performed using a randomly selected sample of 66 citations screened for CL/ P-candidate genes by titles and abstracts. After achieving $a>90 \%$ score for the Cohen's Kappa, all the titles and abstracts of the articles found through the database search were independently examined by two screeners. The full text of the articles not excluded in the above process was manually reviewed, and all results from the screening were recorded in the Primary Excel Workbook. The data collected were displayed as a descriptive narrative. A codebook for data extraction from eligible articles was developed, as described previously [16]. The data elements extracted for the codebook included citation information, study level information (characteristics and results), and quality level information. The quality assessment of each study identified was performed using the Newcastle- Ottawa Scale (NOS), considering the selection criteria of pre- and post- fortification samples, comparability of these groups, and the ascertainment of either the exposure or outcome. NOS assigns a maximum score of 9 points where studies showing $<5$ points have high risk of bias and limitations, with these being excluded from a meta-analysis [17].

\section{Bioinformatics analysis of $\mathrm{CL} / \mathrm{P}$ genes}

Functional enrichment analyses of the candidate genes were conducted using the pathway databases Gene Ontology (GO) and Kyoto Encyclopedia of Genes and Genomes (KEGG) through tools of the database for annotation, visualization, and integrated discovery (DAVID, version 6.8, http://david.ncifcrf.gov) [18]. The enrichment of $\mathrm{CL} / \mathrm{P}$ genes in a pathway or GO term was tested by the hypergeometric test implemented in DAVID. The $p$-values were adjusted by false discovery rate (FDR, $q$-value). The significant pathways were obtained by $q<0.05$ and at least four genes from the list of input genes (CL/P genes) in each pathway included. Hierarchical level 4 was used as the cutoff in order to avoid too general GO terms. The miRNAmediated post-transcriptional regulation of the $\mathrm{CL} / \mathrm{P}$ candidate genes was analyzed by using the following method: first, the miRNA-gene pairs were identified from three computationally predicted miRNA-target gene interaction databases, miRanda, PITA, and TargetScan [19-22]; then, the miRNA-gene pairs that were experimentally proofed were selected through miRTarbase analysis [13]; finally, for each miRNA, the enrichment of CL/P candidate genes was examined using Fisher's Exact Test. To investigate which human phenotype terms are strongly enriched for the candidate genes, the WEB-based Gene SeT AnaLysis Toolkit (WebGestalt) (http://webgestalt.org) was used to perform the overrepresentation enrichment analysis (OEA) using the Human Phenotype Ontology (HPO) annotation database. The top 30 results from the OEA were retrieved on March 15, 2019. The minimum number of genes per category was set to 5 (default). The Benjamini-Hochberg procedure was used for multiple test correction [23]. FDR values were used for all statistical analyses.

\section{Genotype-phenotype association analysis}

Genotype-phenotype association analysis was conducted using GWAS available from dbGaP (dbGaP accession phs000774.v1.p1, https://www.ncbi.nlm.nih.gov/gap). This dbGaP repository includes 11,925 individuals from 4058 families, mostly from trios. Out of 11,925 individuals, 5327 were reported as Caucasians, 2221 as Asians, 473 as Africans, and 3904 were reported as having more than one ancestry. A total of 5008 individuals were reported as of Hispanic ethnicity. Not including the parents, $52 \%$ of children were males and $48 \%$ were females. Array-based genotypes from Illumina Infinium HumanCore Beadchips, including GWAS markers from HumanCore v1 with additional exome and custom contents from the Center for Craniofacial and Dental Genetics (CCDG) consortium, were analyzed with the PLINK software (version 1.90b) to test the association of each directly genotyped variant in the genes listed through the systematic review. Transmission disequilibrium test (TDT) was applied for maximizing analytical power for trios and for minimizing artifacts from population stratification.

\section{Cell culture}

Human lip fibroblasts were obtained from JCRB Cell Bank (\#JCRB9103KD) and cultured in Dulbecco's Modified Eagle 
Medium (DMEM) supplemented with 10\% fetal bovine serum (FBS), penicillin/streptomycin, and L-glutamine. Human lip fibroblasts were plated onto 96-well cell culture plates at a density of 5000/well and treated with a mimic for either negative control, miR-124-3p, miR- 369-3p, miR374a-5p, miR-374b-5p, miR-497-5p, and miR-655-3p (mirVana miRNA mimic, ThermoFisher Scientific) using TransIT-X2 system (Mirus Bio LLC, Madison, WI), following the manufacturer's protocol $(3 \mathrm{pmol}$ mimic and $0.3 \mu \mathrm{l}$ transfection reagent in $100 \mu \mathrm{l}$ DMEM per well). Cell proliferation was determined using a cell counting kit 8 (Dojindo Molecular Technologies, Gaithersburg, MD) ( $n=6$ per group).

\section{Quantitative RT-PCR}

Total RNAs were isolated from cultured human lip fibroblasts treated with a mimic for target miRNAs for 1 day ( $\mathrm{n}=6$ per group), using the QIAshredder and RNeasy mini extraction kit (QIAGEN), as previously described [24]. The PCR primers used for quantitative RT-PCR are listed in Additional file 1: Table S1.

\section{Statistical analysis}

Two-tailed student's $t$ tests were applied for the statistical analysis. A $p$ value $<0.05$ was considered statistically significant. For all graphs, data are represented as mean \pm standard deviation (SD).

\section{Results \\ Literature search}

Our systematic search identified a total of 5016 publications. After eliminating 2653 duplicates from the list, the remaining 2363 articles were further screened, using the titles and abstracts, independently by the two screeners, which resulted in 1558 publications being further excluded based on reasons such as referring to non-genetic studies and case reports. The remaining 748 articles were further assessed for eligibility through manual full-text review. Through this process, 393 articles satisfying all inclusion criteria were selected while 355 articles were excluded. These selected 393 studies were used for collection of $\mathrm{CL} / \mathrm{P}$-candidate genes and in the follow-up analyses (Fig. 1 and Additional file 1: Table S2).

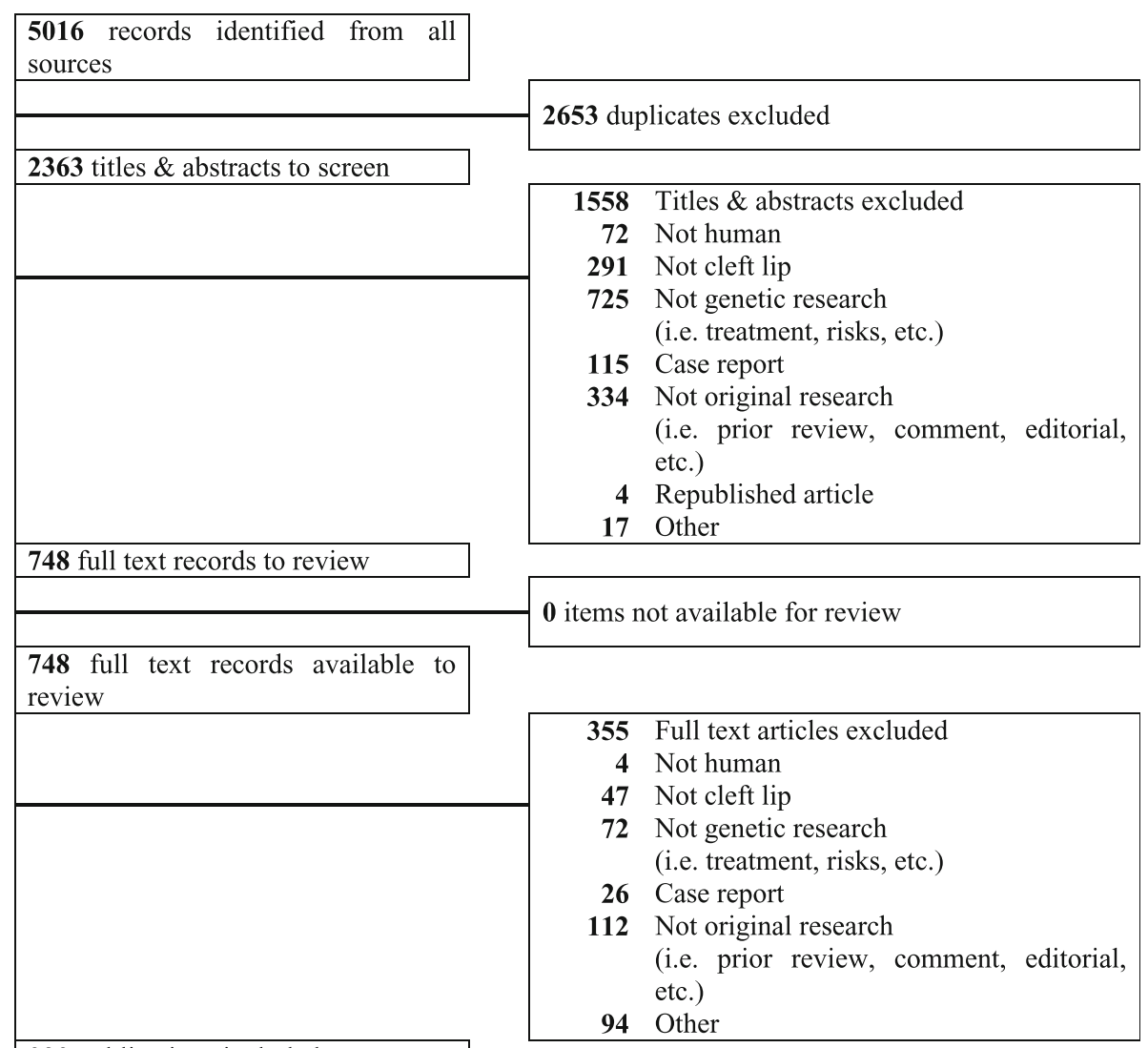

393 publications included Reporting on $\mathbf{3 9 3}$ studies

Fig. 1 PRISMA flowchart for study selection. A graphical representation of the flow of citations reviewed in the course of the systematic review was provided using a PRISMA flow diagram 


\section{Summary of human $\mathrm{CL} / \mathrm{P}$ genes}

We identified $172 \mathrm{CL} / \mathrm{P}$-candidate genes from the qualified studies above (Table 1 and Additional file 1: Tables S3-S8). For the bioinformatics analyses, we excluded phenotypic markers and genes with unknown genomic location. Among the $\mathrm{CL} / \mathrm{P}$-candidate genes, 10 genes were studied at least five times in different populations: IRF6 (52 studies, encoding interferon regulatory factor 6 , located at genomic locus 1q32.2), MTHFR (26 studies, encoding methylenetetrahydrofolate reductase, at 1p36.2), TGFA (18 studies, encoding transforming growth factor alpha, at 2q13.3), MSX1 (25 studies, encoding msh homeobox 1 , at 4p16.2), TGFB3 (16 studies, encoding transforming growth factor beta 3 , at 14q24.3), NECTIN1 (10 studies, encoding Nectin cell adhesion molecule 1, at 11q23.3), BMP4 (10 studies, encoding bone morphogenetic protein 4, at 14q22.2), FOXE1 (6 studies, encoding forkhead box E1, at 9q22.33), BCL3 (6 studies, encoding B-cell CLL/lymphoma 3, at 19q13.32), and CRISPLD2 (5 studies, encoding cysteine rich secretory protein LCCL domain containing 2, at 16q24.1). Most of the gene mutations $(168 / 177=94.9 \%)$ were reported only in non-syndromic $\mathrm{CL} / \mathrm{P}$ while mutations in nine genes (ADH1C, FGFR1, IRF6, MID1, NECTIN1, PHF8, SOX9, TGFA, and TP63) were also reported in syndromic CL/P $(9 / 177=5.1 \%)$. Several genes were reported only in syndromic cases; CHD7 (CHARGE syndrome), CR1 (van der Woude syndrome), EFNB1 (craniofrontonasal syndrome), KISS1R (Kallmann syndrome), MID1 (Opitz G/BBB syndrome), REN (van der Woude syndrome), and SOX9 (Pierre-Robin syndrome). Mutations in eight genes (BCL3, BMP4, IRF6, MSX1, MTHFR, NECTIN1, TGFA, and TGFB3) were reported across different ethnic groups such as Caucasians, African Americans, Hispanics, and Asians, including but not limited to Chinese, Japanese, Indian, Turkish, Polish, Finnish, Brazilian, American, and European. By contrast, a total of 75 genes were reported as insignificant in some populations. For example, while mutations in TGFA were significant in the Iranian, Korean, Caucasian and Chilean population, they were not significant in the South American, Italian, Malaysian or Indian populations. Mutations in IRF6 were not significant in some studies, but they were significant in larger studies in various populations (Additional file 1: Tables S3-S8). Recent advances in the discovery of genetic variants at whole genome level and the design of genome-wide approaches enable investigators to identify the involvement of multiple genes and loci in a single study (e.g., GWAS). Mutations in multiple genes and loci were reported in 87 and 12 studies, respectively. A significant association of IRF6, MSX1 and NECTIN1 with CL/P has been reported in several GWAS in different populations (Additional file 1: Table S4). However, no significant association was reported in several studies conducted in Danish, Mesoamerican, and African populations for IRF6, American and Latvian populations for MSX1, and Taiwanese population for NECTIN1 (Additional file 1: Table S6).

\section{GO term enrichment analysis}

We analyzed CL/P genes enriched in the GO terms to assess the functional features of $\mathrm{CL} / \mathrm{P}$ genes (Additional file 1: Tables S9-S12). The most specific enriched terms among GO biological processes showed strong association with development and morphogenesis of other organs (Additional file 1: Tables S9 and S10). These results suggest that $\mathrm{CL} / \mathrm{P}$ genes may potentially cause additional developmental disorders while $70 \%$ of $\mathrm{CL} / \mathrm{P}$ cases are non-syndromic without any additional birth defects. In non-syndromic CL/P cases, molecules that are specifically expressed in the lip region during lip formation may help explain the molecular mechanism in regard to how only lip formation is affected. Lip formation involves the growth and fusion of maxillary and nasal processes during embryogenesis [25]. We identified a strong association with positive and negative regulators of several cellular processes, including cell proliferation, apoptosis, differentiation, and epithelial-to-mesenchymal transition. We also found that genes involved in folic acid metabolic process were significantly enriched (7 CL/P genes) (Additional file 1: Table S10). The current approach for the prevention of neural tube defects and CL/P is folic acid supplementation [26]. Our results suggest that some individuals with $\mathrm{CL} / \mathrm{P}$ have defects in folic acid metabolism, and that a folic acid supplement can potentially prevent $\mathrm{CL} / \mathrm{P}$.

Among the enriched GO terms in its domain Molecular Function (MF) (Additional file 1: Tables S9 and S11), we observed enrichment of several terms related to molecular binding: transcription factor activity, sequence-specific binding (28 CL/P genes), sequence-specific DNA binding (23 CL/P genes), chromatin binding (16 CL/P genes), and binding to Frizzled, a family of $\mathrm{G}$ protein-coupled receptors for WNT ligands (11 CL/P genes). The remaining enriched terms in the MF domain included: protein homodimerization activity (26 CL/P genes); growth factor activity (13 CL/ $P$ genes), which is induced by FGF, BMP, TGF $\beta$ and PDGF; protein tyrosine kinase activity (10 CL/P genes), 1phosphatidylinositol-3 kinase activity (8 CL/P genes), and phosphatidylinositol-4,5-bisphosphate 3-kinase activity (8 $\mathrm{CL} / \mathrm{P}$ genes) induced by FGF signaling; and others. Thus, the GO MF term analysis highlighted the contribution of morphologic factors involved in WNT, FGF, BMP, TGF , and PDGF signaling pathways.

Among GO terms representing cellular components (Additional file 1: Tables S9 and S12), most terms were enriched in extracellular components: extracellular region (48 CL/P genes), extracellular space (36 CL/P genes), cell surface (27 CL/P genes), and proteinaceous extracellular matrix (ECM) (22 CL/P genes). In GO terms, many 
Table 1 Summary of genes associated with cleft lip with/without cleft palate in humans

\begin{tabular}{|c|c|c|c|}
\hline $\begin{array}{l}\text { Gene } \\
\text { symbol }\end{array}$ & Gene name & Loci & Syndromic/ Nonsyndromic \\
\hline ABCA4 & ATP binding cassette subfamily A member 4 & $1 \mathrm{p} 22.1$ & Nonsyndromic \\
\hline$A B C B 1$ & ATP binding cassette subfamily B member 1 & $7 q 21.12$ & Nonsyndromic \\
\hline ADAM3A & ADAM metallopeptidase domain 3A & $8 p 11.22$ & Nonsyndromic \\
\hline ACSS2 & Acyl-coenzyme A synthetase short-chain family member 2 & $20 q 11.22$ & Nonsyndromic \\
\hline ADAM5 & ADAM metallopeptidase domain 5 & $8 p 11.22$ & Nonsyndromic \\
\hline ADAMTS20 & ADAM metallopeptidase with thrombospondin type 1 motif 20 & $12 q 12$ & Nonsyndromic \\
\hline ADGRL2 & Adhesion G protein-coupled receptor L2 & $1 \mathrm{p} 31.1$ & Nonsyndromic \\
\hline $\mathrm{ADH} 1 \mathrm{C}$ & Alcohol dehydrogenase 1C (class I), gamma polypeptide & $4 q 23$ & $\begin{array}{l}\text { Nonsyndromic \& Syndromic: Fetal } \\
\text { alcohol syndrome }\end{array}$ \\
\hline AQP7 & Aquaporin 7 & $9 p 13.3$ & Nonsyndromic \\
\hline ARHGAP29 & Rho GTPase activating protein 29 & $1 \mathrm{p} 22.1$ & Nonsyndromic \\
\hline ASS1 & Argininosuccinate synthase 1 & $9 q 34.11$ & Nonsyndromic \\
\hline AXIN2 & Axin 2 & $17 q 24.1$ & Nonsyndromic \\
\hline BAG4 & BCL2 associated athanogene 4 & $8 p 11.23$ & Nonsyndromic \\
\hline $\mathrm{BCL} 3$ & B-cell CLL/lymphoma 3 & $19 q 13.32$ & Nonsyndromic \\
\hline BHMT2 & Betaine-homocysteine S-methyltransferase 2 & $5 q 14.1$ & Nonsyndromic \\
\hline BLM & Bloom syndrome RecQ like helicase & $15 q 26.1$ & Nonsyndromic \\
\hline BMP4 & Bone morphogenetic protein 4 & $14 q 22.2$ & Nonsyndromic \\
\hline BMPR1B & Bone morphogenetic protein receptor type 1B & $4 q 22.3$ & Nonsyndromic \\
\hline BRIP1 & BRCA1 interacting protein C-terminal helicase 1 & $17 q 23.2$ & Nonsyndromic \\
\hline CBS & Cystathionine-beta-synthase & $21 q 22.3$ & Nonsyndromic \\
\hline $\mathrm{CDH} 1$ & Cadherin 1 & $16 q 22.1$ & Nonsyndromic \\
\hline $\mathrm{CDH} 2$ & Cadherin 2 & $18 q 12.1$ & Nonsyndromic \\
\hline CENPJ & Centromere protein J & $\begin{array}{l}13 q 12.12- \\
q 12.13\end{array}$ & Nonsyndromic \\
\hline CHD7 & Chromodomain helicase DNA binding protein 7 & $8 q 12.2$ & Syndromic: CHARGE syndrome \\
\hline CLPTM1 & CLPTM1, transmembrane protein & $19 q 13.32$ & Nonsyndromic \\
\hline COL4A2 & Collagen type IV alpha 2 chain & $13 q 34$ & Nonsyndromic \\
\hline COL4A3 & Collagen type IV alpha 3 chain & $2 q 36.3$ & Nonsyndromic \\
\hline COL4A4 & Collagen type IV alpha 4 chain & $2 q 36.3$ & Nonsyndromic \\
\hline COL21A1 & Collagen type XXI alpha 1 chain & $\begin{array}{l}6 \mathrm{p} 12.1 ; 6 \mathrm{p} 12.3- \\
\mathrm{p} 11.2\end{array}$ & Nonsyndromic \\
\hline CR1 & Complement C3b/C4b receptor 1 (Knops blood group) & $1 \mathrm{q} 32.2$ & Syndromic: Van der Woude syndrome \\
\hline CRISPLD2 & Cysteine rich secretory protein LCCL domain containing 2 & $16 q 24.1$ & Nonsyndromic \\
\hline CYP1A1 & Cytochrome P450 family 1 subfamily A member 1 & $15 q 24.1$ & Nonsyndromic \\
\hline CYP2E1 & Cytochrome P450 family 2 subfamily E member 1 & $10 q 26.3$ & Nonsyndromic \\
\hline DCAF7 & DDB1 and CUL4 associated factor 7 & $17 q 23.3$ & Nonsyndromic \\
\hline DHFR & Dihydrofolate reductase & $5 q 14.1$ & Nonsyndromic \\
\hline DICER1 & Dicer 1, ribonuclease III & $14 q 32.13$ & Nonsyndromic \\
\hline DLX1 & Distal-less homeobox 1 & $2 q 31.1$ & Nonsyndromic \\
\hline DMD & Dystrophin & Xp21.2-p21.1 & Nonsyndromic \\
\hline DVL2 & Dishevelled segment polarity protein 2 & 17p13.1 & Nonsyndromic \\
\hline E2F1 & E2F transcription factor 1 & $20 q 11.22$ & Nonsyndromic \\
\hline EFNB1 & Ephrin B1 & $X q 13.1$ & Syndromic: Craniofrontonasal syndrome \\
\hline
\end{tabular}


Table 1 Summary of genes associated with cleft lip with/without cleft palate in humans (Continued)

\begin{tabular}{|c|c|c|c|}
\hline $\begin{array}{l}\text { Gene } \\
\text { symbol }\end{array}$ & Gene name & Loci & Syndromic/ Nonsyndromic \\
\hline EIF2B3 & Eukaryotic translation initiation factor $2 \mathrm{~B}$ subunit gamma & $1 \mathrm{p} 34.1$ & Nonsyndromic \\
\hline EGF61 & Epidermal growth factor 61 & $4 q 25$ & Nonsyndromic \\
\hline ESRRG & Estrogen related receptor gamma & $1 q 41$ & Nonsyndromic \\
\hline EVC & EvC ciliary complex subunit 1 & $4 p 16.2$ & Nonsyndromic \\
\hline EVC2 & EvC ciliary complex subunit 2 & $4 p 16.2$ & Nonsyndromic \\
\hline EYA1 & EYA transcriptional coactivator and phosphatase 1 & $8 q 13.3$ & Nonsyndromic \\
\hline $\mathrm{F} 13 \mathrm{~A} 1$ & Coagulation factor XIII A chain & $6 \mathrm{p} 25.1$ & Nonsyndromic \\
\hline FAM49A & Family with sequence similarity 49 member $A$ & $2 p 24.2$ & Nonsyndromic \\
\hline FGF1 & Fibroblast growth factor 1 & $5 q 31.3$ & Nonsyndromic \\
\hline FGF2 & Fibroblast growth factor 2 & $4 q 28.1$ & Nonsyndromic \\
\hline FGF3 & Fibroblast growth factor 3 & $11 \mathrm{q} 13.3$ & Nonsyndromic \\
\hline FGF10 & Fibroblast growth factor 10 & $5 p 12$ & Nonsyndromic \\
\hline FGF18 & Fibroblast growth factor 18 & $5 q 35.1$ & Nonsyndromic \\
\hline FGFR1 & Fibroblast growth factor receptor 1 & $8 p 11.23$ & $\begin{array}{l}\text { Nonsyndromic \& Syndromic: Kallmann } \\
\text { syndrome }\end{array}$ \\
\hline FGFR2 & Fibroblast growth factor receptor 2 & $10 q 26.13$ & Nonsyndromic \\
\hline FOXE1 & Forkhead box E1 & $9 q 22.33$ & Nonsyndromic \\
\hline FOXF2 & Forkhead box F2 & $6 p 25.3$ & Nonsyndromic \\
\hline FOXG1 & Forkhead box G1 & $14 q 12$ & Nonsyndromic \\
\hline FOXP2 & Forkhead box protein P2 & $7 q 31.1$ & Nonsyndromic \\
\hline FZD6 & Frizzled class receptor 6 & $8 q 22.3$ & Nonsyndromic \\
\hline GABRB3 & Gamma-aminobutyric acid type A receptor beta 3 subunit & $15 q 12$ & Nonsyndromic \\
\hline GAD1 & Glutamate decarboxylase 1 & $2 q 31.1$ & Nonsyndromic \\
\hline $\mathrm{GCH} 1$ & GTP cyclohydrolase 1 & $14 q 22.2$ & Nonsyndromic \\
\hline GLI2 & GLI family zinc finger 2 & $2 q 14.2$ & Nonsyndromic \\
\hline GREM1 & Gremlin 1, DNA family BMP antagonist & $15 q 13.3$ & Nonsyndromic \\
\hline GSTM1 & Glutathione S-transferase mu 1 & $1 \mathrm{p} 13.3$ & Nonsyndromic \\
\hline GSTP1 & Glutathione S-transferase pi 1 & $11 q 13.2$ & Nonsyndromic \\
\hline GSTT1 & Glutathione S-transferase theta 1 & $22 q 11.23$ & Nonsyndromic \\
\hline HECTD1 & HECT domain E3 Ubiquitin protein ligase 1 & $14 q 12$ & Nonsyndromic \\
\hline HKDC1 & Hexokinase domain containing 1 & $10 q 22.1$ & Nonsyndromic \\
\hline IRF6 & Interferon regulatory factor 6 & $1 \mathrm{q} 32.2$ & Nonsyndromic \\
\hline JAG2 & Jagged2 & $14 q 32.33$ & Nonsyndromic \\
\hline JARID2 & Jummonji and AT-rich interaction domain containing 2 & $6 \mathrm{p} 22.3$ & Nonsyndromic \\
\hline $\mathrm{KIF} 2 \mathrm{~A}$ & Kinesin family member $2 \mathrm{~A}$ & $5 q 12.1$ & Nonsyndromic \\
\hline KIF7 & Kinesin family member 7 & $15 q 26.1$ & Nonsyndromic \\
\hline KISS1R & KISS1 receptor & 19p13.3 & Syndromic: Kallmann syndrome \\
\hline KRT18 & Keratin 18 & $12 q 13.13$ & Nonsyndromic \\
\hline LACTB & Lactamase beta & $15 q 22.2$ & Nonsyndromic \\
\hline LHX8 & LIM homeobox 8 & $1 \mathrm{p} 31.1$ & Nonsyndromic \\
\hline LPHN2 & Adhesion G protein-coupled receptor L2 & $1 \mathrm{p} 31.1$ & Nonsyndromic \\
\hline MAFB & MAF bZIP transcription factor B & $20 q 12$ & Nonsyndromic \\
\hline MGAM & Maltase-glucoamylase & $7 q 34$ & Nonsyndromic \\
\hline MID1 & Midline 1 & Xp22.2 & Nonsyndromic \& Syndromic: Opitz G/ \\
\hline
\end{tabular}


Table 1 Summary of genes associated with cleft lip with/without cleft palate in humans (Continued)

\begin{tabular}{|c|c|c|c|}
\hline $\begin{array}{l}\text { Gene } \\
\text { symbol }\end{array}$ & Gene name & Loci & Syndromic/ Nonsyndromic \\
\hline & & & BBB syndrome \\
\hline MMP3 & Matrix metallopeptidase 3 & $11 q 22.2$ & Nonsyndromic \\
\hline MMP9 & Matrix metallopeptidase 9 & $20 q 13.12$ & Nonsyndromic \\
\hline $\mathrm{MROH} 7$ & Maestro heat like repeat family member 7 & $1 p 32.3$ & Nonsyndromic \\
\hline MRPL53 & Mitochondrial ribosomal protein $\mathrm{L} 53$ & $2 \mathrm{p} 13.1$ & Nonsyndromic \\
\hline MSX1 & msh homeobox 1 & $4 p 16.2$ & Nonsyndromic \\
\hline MSX2 & msh homeobox 2 & $5 q 35.2$ & Nonsyndromic \\
\hline MTR & 5-methyltetrahydrofolate-homocysteine methyltransferase & $1 \mathrm{q} 43$ & Nonsyndromic \\
\hline MTRR & 5-methyltetrahydrofolate-homocysteine methyltransferase reductase & $5 p 15.31$ & Nonsyndromic \\
\hline MYH9 & Myosin heavy chain 9 & $22 q 12.3$ & Nonsyndromic \\
\hline MTHFD1 & $\begin{array}{l}\text { Methylenetetrahydrofolate dehydrogenase, cyclohydrolase and } \\
\text { formyltetrahydrofolate synthetase } 1\end{array}$ & $14 q 23.3$ & Nonsyndromic \\
\hline MTHFR & Methylenetetrahydrofolate reductase & 1 p36.22 & Nonsyndromic \\
\hline NAT1 & N-acetyltransferase 1 & $8 p 22$ & Nonsyndromic \\
\hline NAT2 & $\mathrm{N}$-acetyltransferase 2 & $8 p 22$ & Nonsyndromic \\
\hline NECTIN1 & Nectin cell adhesion molecule 1 & $11 q 23.3$ & Nonsyndromic \\
\hline NECTIN2 & Nectin cell adhesion molecule 2 & $19 q 13.32$ & Nonsyndromic \\
\hline NECTIN3 & Nectin cell adhesion molecule 3 & $3 q 13.13$ & Nonsyndromic \\
\hline NOG & Noggin & $17 q 22$ & Nonsyndromic \\
\hline NTN1 & Netrin 1 & 17p13.1 & Nonsyndromic \\
\hline OFC1 & Orofacial cleft 1 & $6 \mathrm{p} 24$ & Nonsyndromic \\
\hline OFC2 & Orofacial cleft 2 & $2 p 14-p 13$ & Nonsyndromic \\
\hline PAH & Phenylalanine hydroxylase & $12 q 23.2$ & Nonsyndromic \\
\hline PARVA & Parvin alpha & $11 p 15.3$ & Nonsyndromic \\
\hline PTCH1 & Patched 1 & $9 q 22.32$ & Nonsyndromic \\
\hline PAX3 & Paired box 3 & $2 q 36.1$ & Nonsyndromic \\
\hline PAX7 & Paired box 7 & 1 p36.13 & Nonsyndromic \\
\hline PAX9 & Paired box 9 & $14 q 13.3$ & Nonsyndromic \\
\hline PCYT1A & Phosphate cytidyly|transferase 1, choline, alpha & $3 q 29$ & Nonsyndromic \\
\hline PDGFC & Platelet derived growth factor $C$ & $4 q 32.1$ & Nonsyndromic \\
\hline PEMT & Phosphatidylethanolamine N-methyltransferase & $17 p 11.2$ & Nonsyndromic \\
\hline PKP1 & Plakophilin 1 & $1 q 32.1$ & Nonsyndromic \\
\hline PHF8 & PHD finger protein 8 & Xp11.22 & Nonsyndromic \\
\hline $\mathrm{PHYH}$ & Phytanoyl-CoA 2-hydroxylase & 10p13 & Nonsyndromic \\
\hline RPS26 & Ribosomal Protein S26 & $12 q 13.2$ & Nonsyndromic \\
\hline PRSS1 & Protease, serine 1 & $7 q 34$ & Nonsyndromic \\
\hline PRSS35 & Protease, serine 35 & $6 q 14.2$ & Nonsyndromic \\
\hline RAD51 & RAD51 recombinase & $15 q 15.1$ & Nonsyndromic \\
\hline RAD54B & RAD54 homolog B & $8 q 22.1$ & Nonsyndromic \\
\hline RARA & Retinoic Acid Receptor Alpha & $17 q 21.2$ & Nonsyndromic \\
\hline RECQL5 & RecQ like helicase 5 & $17 q 25.1$ & Nonsyndromic \\
\hline REG3A & Regenerating family member 3 alpha & $2 \mathrm{p} 12$ & Nonsyndromic \\
\hline REN & Renin & $1 q 32.1$ & Syndromic: Van der Woude syndrome \\
\hline RFC1 & Replication factor C subunit 1 & $4 p 14$ & Nonsyndromic \\
\hline
\end{tabular}


Table 1 Summary of genes associated with cleft lip with/without cleft palate in humans (Continued)

\begin{tabular}{|c|c|c|c|}
\hline $\begin{array}{l}\text { Gene } \\
\text { symbol }\end{array}$ & Gene name & Loci & Syndromic/ Nonsyndromic \\
\hline RHPN2 & Rhophilin Rho GTPase binding protein 2 & $19 q 13.11$ & Nonsyndromic \\
\hline RUNX2 & Runt related transcription factor 2 & $6 \mathrm{p} 21.1$ & Nonsyndromic \\
\hline RYK & Receptor-like tyrosine kinase & $3 q 22.2$ & Nonsyndromic \\
\hline SATB2 & SATB homeobox 2 & $2 q 33.1$ & Nonsyndromic \\
\hline SEC16A & SEC 16 homolog A, endoplasmic reticulum export factor & $9 q 34.3$ & Nonsyndromic \\
\hline SERPINA6 & Serpin family A member 6 & $14 q 32.13$ & Nonsyndromic \\
\hline SLC6A4 & Solute carrier family 6 member 4 & $17 q 11.2$ & Nonsyndromic \\
\hline SMAD1 & SMAD family member 1 & $4 q 31.21$ & Nonsyndromic \\
\hline SMAD2 & SMAD family member 2 & $18 q 21.1$ & Nonsyndromic \\
\hline sox9 & SRY-box 9 & $17 q 24.3$ & Syndromic: Pierre-Robin Syndrome \\
\hline SPRY1 & Sprouty RTK signaling antagonist 1 & $4 q 28.1$ & Nonsyndromic \\
\hline SUMO1 & Small ubiquitin-like modifier 1 & 2q33.1 & Nonsyndromic \\
\hline STK32B & Serine/threonine kinase 32B & $4 p 16.2$ & Nonsyndromic \\
\hline SYNE3 & Spectrin repeat containing nuclear envelop family member 3 & $14 q 32.13$ & Nonsyndromic \\
\hline TAF1B & TATA-box binding protein associated factor, RNA polymerase I subunit B & $2 \mathrm{p} 25.1$ & Nonsyndromic \\
\hline TANC2 & Tetratricopeptide repeat, ankyrin repeat and coiled-coil containing 2 & $17 q 23.2-q 23.3$ & Nonsyndromic \\
\hline TBX22 & T-box 22 & $\mathrm{Xq} 21.1$ & Nonsyndromic \\
\hline TCN2 & Transcobalamin 2 & $22 q 12.2$ & Nonsyndromic \\
\hline TEX11 & Testis expressed 11 & Xq13.1 & Nonsyndromic \\
\hline TFAP2A & Transcription factor AP-2 alpha & $6 \mathrm{p} 24.3$ & Nonsyndromic \\
\hline TGFA & Transforming growth factor alpha & $2 p 13.3$ & Nonsyndromic \\
\hline TGFB1 & Transforming growth factor beta 1 & $2 p 13.3$ & Nonsyndromic \\
\hline TGFB2 & Transforming growth factor beta 2 & $1 q 41$ & Nonsyndromic \\
\hline TGFB3 & Transforming growth factor beta 3 & $14 q 24.3$ & Nonsyndromic \\
\hline TIMP2 & TIMP metallopeptidase inhibitor 2 & $17 q 25.3$ & Nonsyndromic \\
\hline TMEM19 & transmembrane protein 19 & $12 q 21.1$ & Nonsyndromic \\
\hline TNS1 & Tensin 1 & $2 q 35$ & Nonsyndromic \\
\hline TOX3 & TOX high mobility group box family member 3 & $16 q 12.1$ & Nonsyndromic \\
\hline TP63 & Tumor protein p63 & $3 q 28$ & Syndromic: Hay wells or AEC syndrome \\
\hline TPH2 & Tryptophan hydroxylase 2 & $12 \mathrm{q} 21.1$ & Nonsyndromic \\
\hline TPM1 & Tropomyosin 1 (alpha) & $15 q 22.2$ & Nonsyndromic \\
\hline TULP4 & Tubby like protein 4 & $6 q 25.3$ & Nonsyndromic \\
\hline TYMS & Thymidylate synthase & $18 p 11.32$ & Nonsyndromic \\
\hline VAX1 & Ventral anterior homeobox 1 & $10 q 25.3$ & Nonsyndromic \\
\hline $\operatorname{VAX} 2$ & Ventral anterior homeobox 2 & $2 \mathrm{p} 13.3$ & Nonsyndromic \\
\hline WA8 & Von Willebrand factor A domain-containing protein 8 & $13 q 14.11$ & Nonsyndromic \\
\hline WNT3 & Wnt family member 3 & $\begin{array}{l}17 q 21.31- \\
q 21.32\end{array}$ & Nonsyndromic \\
\hline WNT3A & Wnt family member $3 \mathrm{~A}$ & $1 \mathrm{q} 42.13$ & Nonsyndromic \\
\hline WNT5A & Wnt family member $5 \mathrm{~A}$ & $3 p 14.3$ & Nonsyndromic \\
\hline WNT5B & Wnt family member $5 B$ & $12 p 13.33$ & Nonsyndromic \\
\hline WNT6 & Wnt family member 6 & $2 q 35$ & Nonsyndromic \\
\hline WNT9B & Wnt family member 9B & $17 q 21.32$ & Nonsyndromic \\
\hline WNT10A & Wnt family member $10 \mathrm{~A}$ & $2 q 35$ & Nonsyndromic \\
\hline
\end{tabular}


Table 1 Summary of genes associated with cleft lip with/without cleft palate in humans (Continued)

\begin{tabular}{llll}
\hline $\begin{array}{l}\text { Gene } \\
\text { symbol }\end{array}$ & Gene name & Loci & Syndromic/ Nonsyndromic \\
\hline WNT11 & Wnt family member 11 & $11 \mathrm{q} 13.5$ & Nonsyndromic \\
YOD1 & TOD1 deubiquitinase & $1 \mathrm{q} 32.1$ & Nonsyndromic \\
ZNF385B & Zinc finger protein 385B & $2 \mathrm{q} 31.2-\mathrm{q} 31.3$ & Nonsyndromic \\
\hline
\end{tabular}

Due to space limits, the details (e.g. publication ID) of each gene were not provided in this table

growth factors including WNT, FGF, BMP, TGF $\beta$, and PDGF and their extracellular regulators (e.g. ECM) were identified in these groups. These findings are in agreement with the fact that growth factors are activated in the extracellular space.

\section{Human phenotype ontology enrichment analysis}

We used the HPO database to investigate which human phenotype terms were enriched for the identified set of $\mathrm{CL} / \mathrm{P}$ candidate genes. The most enriched phenotypic term was abnormal number of teeth (28 genes), followed by CL (23 genes), cleft upper lip (22 genes), reduced number of teeth ( 25 genes), and oral cleft (36 genes) (Additional file 1: Table S13). Among the top 30 results, most phenotypes were directly related to $\mathrm{CL} / \mathrm{P}$, congenital dental disorders, or congenital abnormalities of the fingers.

\section{KEGG pathway analysis}

We hypothesized that CL/P genes share common features among wide arrays of biological functions and pathways. We therefore examined which biological pathways were enriched with $\mathrm{CL} / \mathrm{P}$ genes by using the DAVID bioinformatics tool and the canonical pathways from the KEGG database (Additional file 1: Table S14). The regulatory pathway annotation was performed based on the score and visualization of the pathways used in the KEGG database. Among the cellular functions in KEGG pathways, 19 pathways were statistically significant in the enrichment of $\mathrm{CL} / \mathrm{P}$ genes $(\mathrm{FDR}<5 \%)$. Seven of these pathways were related to cancer: pathways in cancer (43 CL/P genes), basal cell carcinoma (16 CL/P genes), proteoglycans in cancer $(16 \mathrm{CL} / \mathrm{P}$ genes), melanoma (10 CL/P genes), pancreatic cancer (8 $\mathrm{CL} / \mathrm{P}$ genes), bladder cancer (6 CL/P genes), and colorectal cancer (7 CL/P genes). Previous population-based studies suggest that individuals with $\mathrm{CL} / \mathrm{P}$ have a higher risk of cancer in the breast, brain, and lung later in life [27-29]. Among them, molecules related to Hippo, FGF, WNT, and TGF $\beta$ signaling are highlighted in these cancer-related pathways. These cellular signaling pathways were significantly involved in CL/P: Hippo signaling pathway (22 CL/P genes), signaling pathways regulating pluripotency of stem cells (21 CL/P genes), phosphoinositide 3-kinase (PI3K)Akt signaling pathway (15 CL/P genes), mitogen-activated protein kinase (MAPK) signaling pathway (13 CL/P genes), WNT signaling pathway (11 CL/P genes), and TGF- $\beta$ signaling pathway (9 CL/P genes).

\section{Genotype-phenotype association analysis}

To determine the contribution of genetic variations to $\mathrm{CL} / \mathrm{P}$, a genotype-phenotype association analysis was conducted using GWAS for the genetics of orofacial clefts and related phenotypes (e.g. CL/P) available from dbGaP (dbGaP accession phs000774.v1.p1). By PLINK analysis, we identified 2975 cases vs 8751 controls out of 11,925 individuals. We investigated whether single nucleotide polymorphisms (SNPs) mapped to the CL/ $\mathrm{P}$-candidate genes are associated with human $\mathrm{CL} / \mathrm{P}$ phenotypes using TDT of The Genetics of Orofacial Clefts and Related Phenotypes GWAS dataset. This method is appropriate for statistical imbalances between transmitted and non-transmitted alleles in parent-child trios. We investigated all directly genotyped (i.e. not imputed) variants within the genes found in the systematic review. A total of 5437 variants from 179 genes were included in the association analysis, while some genes did not have the genotyped SNPs in this dataset and some SNPs did not present any variations in the analyzed samples. Because most SNPs in the same gene have strong linkage disequilibrium, we set our candidate-wise significance threshold at $2.79 \times 10^{-4}$ by using the Bonferroni level with the number of genes tested $(0.05 / 179)$. We identified 56 SNPs from 12 genes with $p$-values smaller than $2.79 \times 10^{-4}$ (Table 2). The top association signals were from IRF6 and NTN1 (Netrin 1), which also showed nominal genome-wide significance in the GWAS dataset $\left(p<5 \times 10^{-8}\right)$. We also observed gene-wide significant signals $\left(p<2.5 \times 10^{-6}\right)$ from $A B C A 4$ (ATP-binding cassette, sub-family A, member 4) and $P A X 7$ (paired box 7). Although $A D A M 3 A$ (ADAM metallopeptidase domain 3A), FOXE1 (forkhead box E1), MSX2 (msh homeobox 2), MTHFR (methylenetetrahydrofolate reductase), TP63 (tumor protein p63), TPM1 (tropomyosin 1), VAX1 (ventral anterior homeobox 1), and WNT9B (wingless-type MMTV integration site family, member 9B) did not reach the genome-wide nor gene-wide significance thresholds, these genes reached candidate-wise significance level $\left(p<2.79 \times 10^{-4}\right)$.

\section{Environmental and epigenetic factors}

In addition to gene mutations, genetic background such as ethnicity, population and gender have substantial influence on the birth prevalence of CL/P. Environmental factors such as maternal age, smoking, alcohol consumption, obesity, 
Table 2 Genotype-phenotype association

\begin{tabular}{llll}
\hline Top marker & Type & Gene & $p$-value \\
\hline rs1044516 & UTR3 & IRF6 & $2.66 \times 10^{-9}$ \\
rs1880646 & Intron & NTN1 & $3.02 \times 10^{-9}$ \\
rs4147827 & Intron & ABCA4 & $8.51 \times 10^{-7}$ \\
rs742071 & Intron & PAX7 & $2.09 \times 10^{-6}$ \\
rs6474148 & Intron & ADAM3A & $3.26 \times 10^{-6}$ \\
rs1443435 & UTR3 & FOXE1 & $1.39 \times 10^{-5}$ \\
rs6585429 & Intron & VAX1 & $2.28 \times 10^{-5}$ \\
rs2066462 & Silent & MTHFR & $7.38 \times 10^{-5}$ \\
rs3803499 & Intron & TPM1 & $1.91 \times 10^{-4}$ \\
rs1515497 & Intron & TP63 & $2.19 \times 10^{-4}$ \\
rs14459 & UTR3 & MSX2 & $2.34 \times 10^{-4}$ \\
rs4968282 & Intron & WNT9B & $2.65 \times 10^{-4}$ \\
\hline
\end{tabular}

and micronutrient deficiencies are known to be strong susceptibility risk factors. Recent studies suggest that environmental factors can interact with the epigenetic system and control gene expression at the post- transcriptional level [30-32]. To explore the degree to which miRNAs regulate the expression of CL/P genes, we performed a bioinformatics analysis to identify miRNAs whose target genes are statistically enriched with CL/P genes (Table 3). By applying the adjusted $p$-value (FDR) $<0.1$, a total of 16 miRNAs were significantly enriched with the targeted CL/P genes. These 16 miRNAs were categorized into 10 known and one unknown miRNA families: the miR-27 family (hsa-miR- 27b3p, 23 CL/P genes), miR-124 family (hsa- miR- 124-3p, 29 CL/P genes), miR-154 family (hsa-miR-369-3p, $13 \mathrm{CL} / \mathrm{P}$ genes; hsa- miR-655-3p, $14 \mathrm{CL} / \mathrm{P}$ genes; hsa-miR-300, 18 CL/P genes; hsa-miR-381-3p, 18 CL/P genes), miR-203 family (hsa-miR-203a-3p, 9 CL/P genes), miR-368 family (hsa-miR-376b-3p, $10 \mathrm{CL} / \mathrm{P}$ genes), miR-374 family (hsa-miR-

Table 3 MicroRNA (miRNA) enrichment analysis of 161 human $C L / P$ genes $(F D R<0.1)$

\begin{tabular}{|c|c|c|c|}
\hline $\begin{array}{l}\text { miRNA } \\
\text { family ID }\end{array}$ & miRNA name & Gene symbol & $\begin{array}{l}\text { FDR } \\
(\mathrm{BH})\end{array}$ \\
\hline miR-154 & $\begin{array}{l}\text { hsa-miR- } \\
369-3 p\end{array}$ & CHD7, DMD, EYA1, GABRB3, GREM1, HOXB3, NOG, PAX6, TIMP2, TULP4, BAG4, FZD6, YOD1 & 0.03 \\
\hline miR-154 & $\begin{array}{l}\text { hsa-miR- } \\
655-3 p\end{array}$ & BCL2, DMD, EN2, GREM1, HOXB3, MAFB, MID1, NTN1, PAX6, SATB2, TULP4, CYP1A1, FZD6, YOD1 & 0.03 \\
\hline miR-374 & $\begin{array}{l}\text { hsa-miR- } \\
374 a-5 p\end{array}$ & $\begin{array}{l}\text { CHD7, DMD, EYA1, FGFR2, FOXG1, HECTD1, HOXB3, JARID2, MSX1, NOG, NTN1, PAX6, RAD51, RHPN2, RUNX2, TGFA, } \\
\text { TNS1, WNT5A, WNT5B, YOD1, EN2, FZD6 }\end{array}$ & 0.07 \\
\hline miR-374 & $\begin{array}{l}\text { hsa-miR- } \\
\text { 374b-5p }\end{array}$ & $\begin{array}{l}\text { CHD7, DMD, EYA1, FGFR2, FOXG1, HECTD1, HOXB3, JARID2, MSX1, NOG, NTN1, PAX6, RAD51, RHPN2, RUNX2, TGFA, } \\
\text { TNS1, WNT5A, WNT5B, YOD1, EN2, FZD6 }\end{array}$ & 0.07 \\
\hline miR-497 & $\begin{array}{l}\text { hsa-miR- } \\
497-5 p\end{array}$ & $\begin{array}{l}\text { AXIN2, BAG4, BCL2, CHD7, CRISPLD2, EN2, EYA1, FGF1, FGF2, FGFR1, FGFR2, FOXP2, FZD6, HECTD1, JARID2, PAX7, } \\
\text { RHPN2, SATB2, SLC6A4, WNT3A, YOD1, MTHFR, RUNX2, TFAP2A, TPM1 }\end{array}$ & 0.07 \\
\hline miR-124 & $\begin{array}{l}\text { hsa-miR- } \\
124-3 p\end{array}$ & $\begin{array}{l}\text { GABRB3, GCH1, JAG2, KIF2A, MTR, MYH9, ROR2, RYK, TANC2, ARHGAP29, COL4A4, DVL2, EFNB1, FGF1, FGFR1, FOXF2, } \\
\text { GREM1, HOXB3, KIF7, PDGFC, PEMT, PKP1, RAD51, RUNX2, SOX9, TBX22, TP63, TPM1, WNT5B }\end{array}$ & 0.09 \\
\hline miR-1271 & $\begin{array}{l}\text { hsa-miR- } \\
1271-3 p\end{array}$ & $C B S, D C A F 7, F Z D 6, R A D 51, Y O D 1$ & 0.09 \\
\hline miR-203 & $\begin{array}{l}\text { hsa-miR- } \\
\text { 203a-3p }\end{array}$ & E2F1, EN2, GREM1, HECTD1, KIF2A, PAX6, RUNX2, SMAD2, TP63 & 0.09 \\
\hline miR-27 & $\begin{array}{l}\text { hsa-miR- } \\
\text { 27b-3p }\end{array}$ & $\begin{array}{l}\text { BCL3, CHD7, COL21A1, DVL2, EN2, EYA1, FGF1, FOXP2, GABRB3, GCH1, GREM1, HOXB3, PAX9, RARA, SATB2, SMAD1, } \\
\text { STK32B, SMAD2, DCAF7, PAX3, PAX7, SEC16A, WNT9B }\end{array}$ & 0.09 \\
\hline miR-154 & hsa-miR-300 & $\begin{array}{l}\text { CR1, DVL2, FGF1, FGFR2, FOXF2, FOXP2, GABRB3, GAD1, HECTD1, JAG2, MID1, PDGFC, TANC2, TGFB3, WNT5A, } \\
\text { CRISPLD2, GREM1, PHF8 }\end{array}$ & 0.09 \\
\hline NA & $\begin{array}{l}\text { hsa-miR- } \\
\text { 3678-3p }\end{array}$ & E2F1, GABRB3, JARID2, SLC6A4, YOD1 & 0.09 \\
\hline miR-368 & $\begin{array}{l}\text { hsa-miR- } \\
\text { 376b-3p }\end{array}$ & CRISPLD2, EN2, FGFR1, MYH9, PAX3, PAX6, PRSS35, TANC2, FGF2, FOXG1 & 0.09 \\
\hline miR-154 & $\begin{array}{l}\text { hsa-miR- } \\
381-3 p\end{array}$ & $\begin{array}{l}\text { CR1, DVL2, FGF1, FGFR2, FOXF2, FOXP2, GABRB3, GAD1, HECTD1, JAG2, MID1, PDGFC, TANC2, TGFB3, WNT5A, } \\
\text { CRISPLD2, GREM1, PHF8 }\end{array}$ & 0.09 \\
\hline miR-503 & $\begin{array}{l}\text { hsa-miR- } \\
503-5 p\end{array}$ & HECTD1, JARID2, MAFB, WNT3A, BCL2, DHFR, FGF2, FGFR1, SMAD2, TFAP2A & 0.09 \\
\hline miR-550 & $\begin{array}{l}\text { hsa-miR- } \\
\text { 550a-3-5p }\end{array}$ & CBS, DCAF7, FZD6, RAD51, YOD1 & 0.09 \\
\hline miR-550 & $\begin{array}{l}\text { hsa-miR- } \\
\text { 550a-5p }\end{array}$ & $C B S, D C A F 7, F Z D 6, R A D 51$, YOD1 & 0.09 \\
\hline
\end{tabular}


374a-5p, 22 CL/P genes; hsa-miR-374b-5p, 22 CL/P genes), miR-497 family (hsa-miR-497-5p, 25 CL/P genes), miR-503 family (hsa-miR-503-5p, $10 \mathrm{CL} / \mathrm{P}$ genes), miR-550 family (hsa-miR-550a-5p, 5 CL/P genes; hsa-miR-550a-3-5p, 5 CL/ $\mathrm{P}$ genes), miR-1271 family (hsa-miR-1271-3p, $5 \mathrm{CL} / \mathrm{P}$ genes), and an unknown family (hsa-miR-3678-3p, $5 \mathrm{CL} / \mathrm{P}$ genes). Notably, except for hsa-miR-27b-3p [33], these miRNAs have not yet been reported in $\mathrm{CL} / \mathrm{P}$. In the enrichment analysis, $\mathrm{CL} / \mathrm{P}$ genes regulated by multiple miRNAs were: EN2 (targeted by 6 miRNA families), FZD6 (targeted by 5 miRNA families), HECTD1 (targeted by 5 miRNA families), and YOD1 (targeted by 5 miRNA families) (Table 4). Because gene expression of EN2, FZD6, HECTD1, and YOD1 is regulated by several miRNAs, expression of these genes may be more susceptible to environmental factors during lip formation.

\section{Experimental validation}

miRNA regulates expression of its genes anti- correlationally [34]. To test whether induction of potential CL/P-candidate miRNAs caused proliferation defects through the suppression of target gene expression, we treated cultured human lip fibroblasts with each miRNA mimic. The miR-497-5p and miR-655-3p mimics were most significantly inhibited cell proliferation in human lip fibroblasts (Fig. 2a). To identify target genes regulated by either miR-497-5p or miR-655-3p, we performed quantitative RT-PCR analyses for the predicted target genes (AXIN2, BAG4, BCL2, CHD7, CRISPLD2, EN2, EYA1, FGF1, FGF2, FGFR1, FGFR2, FOXP2, FZD6, HECTD1, JARID2, MTHFR, PAX7, RHPN2, RUNX2, SATB2, SLC6A4, TFAP2A, TPM1, WNT3A, and YOD1 for miR-497-5p; and BCL2, CYP1A1, DMD, EN2, FZD6, GREM1, HOXB3, MAFB, MID1, NTN1, PAX6, SATB2, TULP4, and YOD1 for miR-655-3p) in human lip fibroblasts after treatment with mimics of either miR-497-5p or miR-655-3p. The expression of almost target genes except EN2, GREM1, MAF6, TULP4 and YOD1 was significantly downregulated by treatment with miR- 655-3p mimic (Fig. 2b). The expression of target genes (BAG4, CHD7, CRISPLD2, FGFR1, FOXP2, HECTD1, RUNX2, and TFAP2A) was significantly downregulated by treatment with miR-497-5p mimic (Fig. 2c). PAX6 was excluded because its expression is restricted in the anterior ectoderm during early embryogenesis and the ectoderm of craniofacial surface during craniofacial development [35-37]. EYA1 expression was unexpectedly increased after treatment with miR-497-5p mimic.

\section{Discussion}

Orofacial cleft is described in approximately 400 known human syndromes [38-40]. Several factors have been implicated in clefting by studies of mouse models [25] and genetic screening in humans. Our literature search identified 177 genes as possible causative genes of CL/P. Our follow-up bioinformatics analysis could group genes by common features of $\mathrm{CL} / \mathrm{P}$ genes in function, pathway, and miRNA regulation. As expected, the contribution of most of the highlighted pathways (e.g. FGF, Hippo, TGF $\beta$, and WNT) to the growth and developmental process has been shown in previous mouse genetic studies for craniofacial development [25]. Cellular metabolic pathways such as one-carbon metabolism are also highlighted in KEGG pathway analysis. The one-carbon metabolism, mediated by the folate cofactor, is involved in multiple physiological processes including biosynthesis of purines and thymidine, amino acid homeostasis, epigenetic maintenance, and redox defense [41]. Mice with one-carbon metabolic aberrations (Mthfd1 ${ }^{-/-}$ mice) are embryonic lethal by E12.5, with craniofacial deformities including midfacial cleft [41]. However, in human studies it is still controversial in the significance of genetic mutations in genes involved in one-carbon metabolism [42-45]; further studies are necessary to reach to the conclusive evidence.

Multiple processes synthesize miRNAs and then transcribe them as long primary transcripts that are cleaved by Dicer, a type 3 ribonuclease, to produce mature, functional miRNAs. In human genetic studies, the increasing number of studies show functional significance of single- nucleotide polymorphisms (SNPs) in genes related to CL/P [46-50]. These SNPs might alter the binding activity of miRNAs. For example, previous studies show that SNPs in the miRNA-binding sites of MSX1, FGF2, FGF5 and FGF9 are associated with the susceptibility of nonsyndromic orofacial clefts [51, 52]. A recent study shows that plasma miRNAs are differentially expressed in nonsyndromic CP and nonsyndromic CL/P [53]. The miRNAs may also systemically regulate gene expression during embryogenesis, while some miRNAs may uniquely regulate gene expression in some particular tissues with an interaction with mRNAs expressed in a tissue-specific manner. In mice, loss of Dicer results in absence of mature miRNAs and, therefore, the phenotype in Dicer knockout mice reflects how important miRNAs are for proper development. Interestingly, cranial neural crest $(\mathrm{CNC})$ cell- specific Dicer knockout (Dicer ${ }^{F / F}$;Wnt1-Cre) mice, but not epithelial-specific Dicer knockout $\left(\right.$ Dicer $^{F / F} ;$ K14-Cre) mice, exhibit severe midfacial deformities resulting from decreased cell proliferation and increased apoptosis in the developing craniofacial regions [54-57], indicating that miRNAs have crucial roles in the fate determination of $\mathrm{CNC}$ cells during midfacial development [54-57]. In this study, to evaluate the function of each miRNA in cell proliferation/survival, we employed human lip fibroblasts, CNC-derived cells, for our experimental validation. The functional significance of candidate miRNAs were tested 
Table 4 Human CL/P genes targeted by at least two microRNA (miRNA) families

\begin{tabular}{|c|c|c|}
\hline Gene & \# miRNA families & miRNA family \\
\hline EN2 & 6 & miR-154, miR-203, miR-27, miR-368, miR-374, miR-497 \\
\hline YOD1 & 5 & miR-1271, miR-154, miR-374, miR-497, miR-550 \\
\hline FZD6 & 5 & miR-1271, miR-154, miR-374, miR-497, miR-550 \\
\hline HECTD1 & 5 & miR-154, miR-203, miR-374, miR-497, miR-503 \\
\hline GREM1 & 4 & miR-124, miR-154, miR-203, miR-27 \\
\hline HOXB3 & 4 & miR-124, miR-154, miR-27, miR-374 \\
\hline PAX6 & 4 & miR-154, miR-203, miR-368, miR-374 \\
\hline RAD51 & 4 & miR-124, miR-1271, miR-374, miR-550 \\
\hline CHD7 & 4 & miR-154, miR-27, miR-374, miR-497 \\
\hline EYA1 & 4 & miR-154, miR-27, miR-374, miR-497 \\
\hline RUNX2 & 4 & miR-124, miR-203, miR-374, miR-497 \\
\hline FGF1 & 4 & miR-124, miR-154, miR-27, miR-497 \\
\hline FGFR1 & 4 & miR-124, miR-368, miR-497, miR-503 \\
\hline GABRB3 & 3 & miR-124, miR-154, miR-27 \\
\hline FGFR2 & 3 & miR-154, miR-374, miR-497 \\
\hline JARID2 & 3 & miR-374, miR-497, miR-503 \\
\hline CRISPLD2 & 3 & miR-154, miR-368, miR-497 \\
\hline FOXP2 & 3 & miR-154, miR-27, miR-497 \\
\hline TANC2 & 3 & miR-124, miR-154, miR-368 \\
\hline DVL2 & 3 & miR-124, miR-154, miR-27 \\
\hline DCAF7 & 3 & miR-1271, miR-27, miR-550 \\
\hline$B C L 2$ & 3 & miR-154, miR-497, miR-503 \\
\hline SATB2 & 3 & miR-154, miR-27, miR-497 \\
\hline FGF2 & 3 & miR-368, miR-497, miR-503 \\
\hline SMAD2 & 3 & miR-203, miR-27, miR-503 \\
\hline$D M D$ & 2 & miR-154, miR-374 \\
\hline WNT5A & 2 & miR-154, miR-374 \\
\hline NOG & 2 & miR-154, miR-374 \\
\hline NTN1 & 2 & miR-154, miR-374 \\
\hline FOXG1 & 2 & miR-368, miR-374 \\
\hline RHPN2 & 2 & miR-374, miR-497 \\
\hline WNT5B & 2 & miR-124, miR-374 \\
\hline$J A G 2$ & 2 & miR-124, miR-154 \\
\hline FOXF2 & 2 & miR-124, miR-154 \\
\hline PDGFC & 2 & miR-124, miR-154 \\
\hline$C B S$ & 2 & miR-1271, miR-550 \\
\hline BAG4 & 2 & miR-154, miR-497 \\
\hline MAFB & 2 & miR-154, miR-503 \\
\hline PAX7 & 2 & miR-27, miR-497 \\
\hline WNT3A & 2 & miR-497, miR-503 \\
\hline TFAP2A & 2 & miR-497, miR-503 \\
\hline TPM1 & 2 & miR-124, miR-497 \\
\hline GCH1 & 2 & miR-124, miR-27 \\
\hline KIF2A & 2 & miR-124, miR-203 \\
\hline
\end{tabular}


Table 4 Human CL/P genes targeted by at least two microRNA (miRNA) families (Continued)

\begin{tabular}{lll}
\hline Gene & \# miRNA families & miRNA family \\
\hline MYH9 & 2 & miR-124, miR-368 \\
TP63 & 2 & miR-124, miR-203 \\
PAX3 & 2 & miR-27, miR-368 \\
\hline
\end{tabular}

in cell proliferation/survival assays in cultured human lip fibroblasts. We found that miR-369-3p, miR-655-3p, miR-374a-5p, miR-374b-5p, and miR-497-5p that were first identified in this study as candidates involved in human CL/P suppressed cell proliferation in cultured human lip fibroblasts. Top two candidate miRNAs, miR-655-3p and miR-497-5p, were further tested in the miRNA-gene regulation assay. We found that these miRNA mimics suppressed expression of genes associated with human $\mathrm{CL} / \mathrm{P}$. Thus, the miRNAs predicted were successfully validated in our experiments. Taken together, this study provides a better understanding of $\mathrm{CL} / \mathrm{P}$ as well as data that will be available for the future research of genetic approaches used to characterize individual or cluster miRNAs identified in this study.

In this study, we validated that overexpression of miR-655-3p and miR-497-5p suppressed the expression of multiple genes (BCL2, CYPLA1, DMD, FZD6, HOXB3, MID1, NTN, and SATB2 by miR-655-3p; and BAG4, CHD7, FGFR1, FOXP2, HECTD1, RUNX2, and TFAP2A by

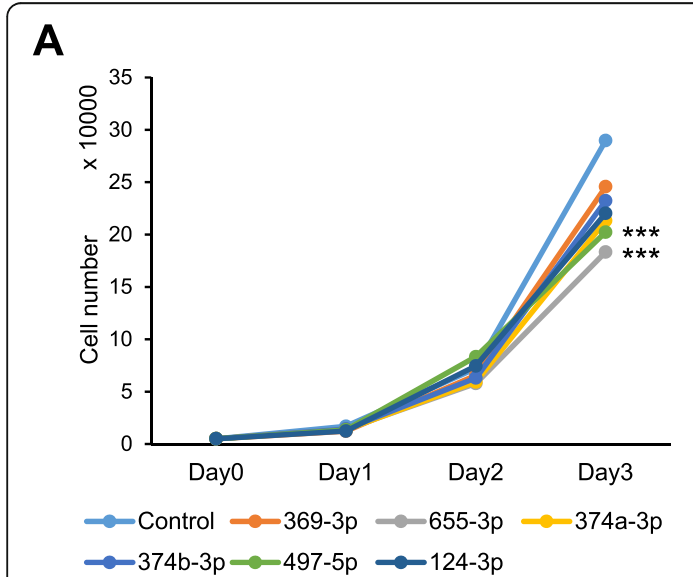

C

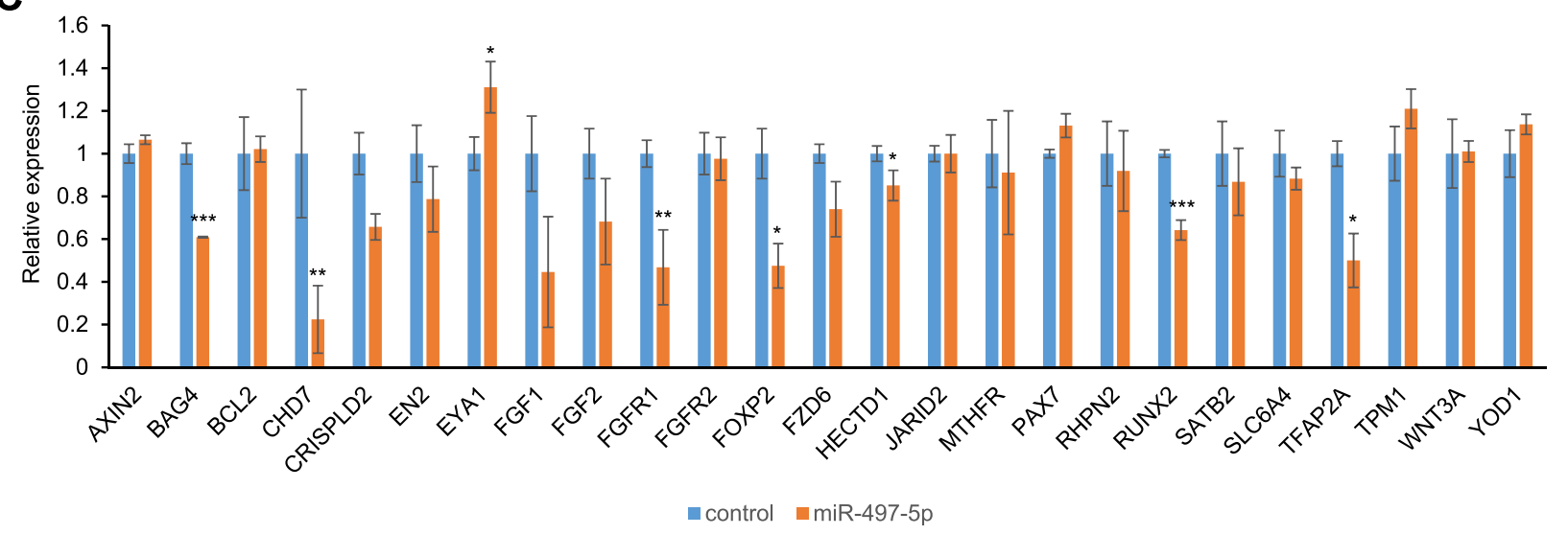

Fig. 2 Experimental validation of predicted miRNAs. a Cell proliferation assays in human lip fibroblasts treated with the indicated mimics of miRNAs. Negative control (control, light blue), miR-369-3p (orange), miR-655-3p (gray), miR-374a-3p (yellow), miR-374b-3p (blue), miR497-5p (light green), and miR-124-3p (dark blue). ${ }^{* * *} p<0.001$. b Quantitative RT-PCR for the indicated genes after treatment with negative control (light blue) or miR-655-3p mimic (orange). ${ }^{*} p<0.05,{ }^{* *} p<0.01,{ }^{* * *} p<0.001$. c Quantitative RT-PCR for the indicated genes after treatment with negative control (light blue) or miR-497-5p mimic (orange). ${ }^{*} p<0.05,{ }^{* *} p<0.01,{ }^{* * *} p<0.001$

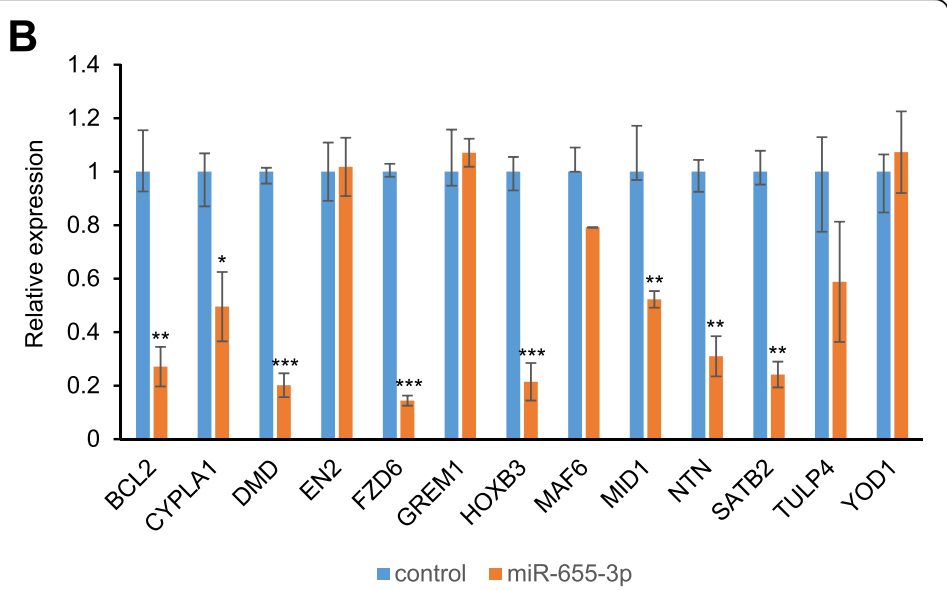

B 
miR-497-5p) that are associated with CL/P. Previous studies indicate that miR-655-3p is downregulated in several cancers through dysregulation of ADAM10 (a disintegrin and metalloproteinase domain-containing protein 10) and the $\mathrm{WNT} / \beta$-catenin pathway $[58,59]$. By contrast, it remains unknown how miR-655-3p expression is induced.

Previous studies also show that miR-497-5p is downregulated in several cancers [60-62]. Interestingly, miR-497-5p is upregulated during myofibroblast differentiation of lung resident mesenchymal stem cells and in the lung tissues of a pulmonary fibrosis mouse model [63]. While it remains unclear what factors and triggers induce miR-655-3p expression, increased miR-655-3p levels may result in accelerated tissue differentiation with less proliferation in various tissues. Several environmental risk factors for $\mathrm{CL} / \mathrm{P}$ such as smoking, alcohol consumption and toxins [64] may cause $\mathrm{CL} / \mathrm{P}$ through the upregulation of these $\mathrm{CL} / \mathrm{P}$-associated miRNAs. In addition, when the levels of these microRNAs are increased too much, multiple $\mathrm{CL} / \mathrm{P}$ genes would be suppressed, as we demonstrated in this study. Our results may partially explain why individuals with $\mathrm{CL} / \mathrm{P}$ show suppression of multiple $\mathrm{CL} / \mathrm{P}$ genes without genetic mutations in the coding regions, which contributes to the complexity of the CL/P etiology.

In this study, we found that miR-497-5p suppressed CHD7 expression at maximum degree of $80 \%$ in cultured human lip fibroblasts. Mutations in CHD7 cause CHARGE syndrome, which is characterized by CL/P (in $20-36 \%$ of the cases), abnormal middle and external ear, coloboma, choanal atresia and hypoplastic semi-circular canals, rhombencephalic dysfunction, hypothalamo- hypophyseal dysfunction, mental retardation, and tracheoesophageal fistula, but do not contribute to nonsyndromic CL/P [65]. miR497-5p also suppressed RUNX2 expression; SNPs in RUNX2 are known to increase the risk of nonsyndromic $\mathrm{CL} / \mathrm{P}$. Although FOXP2 and BAG4 mutations are associated with nonsyndromic $\mathrm{CL} / \mathrm{P}[66,67]$, less is known about the role of these genes in $\mathrm{CL} / \mathrm{P}$ development. Mouse genetic mutant models are useful tools to investigate the role of genes in lip formation, but the loss of these $\mathrm{CL} / \mathrm{P}$ genes fails to cause $\mathrm{CL} / \mathrm{P}$ in mice, although it has not been examined whether suppression of multiple $\mathrm{CL} / \mathrm{P}$-associated genes causes CL/P. However, mice deficient for Tfap $2 a$, which is suppressed by miR-497-5p, exhibit midfacial cleft [68], suggesting that TFAP2A may be a principal downstream target of miR-497-5p in human lip fibroblasts. Mutations in TFAP2A cause Branchio-Oculo-Facial Syndrome (BOFS), which is characterized by $\mathrm{CL} / \mathrm{P}$, branchial arch defects, and ocular anomalies [69], and are associated with nonsyndromic CL/P in several populations. In addition, Tfap $2 a$ deficiency suppresses $F g f 8$ expression in craniofacial regions in mice [68], suggesting that FGF signaling is a downstream pathway of TFAP2A. In this study, we found that
miR-497-5p suppressed FGFR1 gene expression in cultured human lip fibroblasts. Therefore, a combined downregulation of TFAP2A and FGFR1 may compromise this cascade. FGFR1 mutations are also associated with increased risk of nonsyndromic CL/P, as well as of Kallmann syndrome with CL/P [70] and Hartsfield syndrome, which is characterized by holoprosencephaly, ectrodactyly, and $\mathrm{CL} / \mathrm{P}[71]$.

miR-655-3p also suppressed the expression of $F Z D 6$, a WNT receptor, with a maximum reduction of $85 \%$, and of SATB2 in cultured human lip fibroblasts. Mutations in FZD6 are found in nonsyndromic $\mathrm{CL} / \mathrm{P}$ individuals, and SNPs in SATB2 are associated with nonsyndromic $\mathrm{CL} / \mathrm{P}$ in several populations. Importantly, mice deficient for Satb2 also show CL and CP, as seen in individuals with mutations in SATB2. Interestingly, wild-type mouse embryos maternally exposed to phenytoin, a drug used to prevent and control seizures but presenting congenital anomalies as common side effects, show decreased Satb2 expression in craniofacial tissues [72]. This suggests that miR-655-3p may be upregulated in this condition of CL/P. miR-655-3p also suppressed expression of $D M D$ and MID1 in cultured human fibroblasts. These genes are located on the $\mathrm{X}$ chromosome so that their mutations are associated with $\mathrm{X}$-linked nonsyndromic $\mathrm{CL} / \mathrm{P}$ in men and women. Mutations in MID1 are associated with the X-linked Opitz G/BBB syndrome, characterized by midline defects such as $\mathrm{CL}$ and $\mathrm{CP}$, hypertelorism, and laryngo-trachea-esophageal (LTE) abnormalities [73, 74]. CYP1A1 is involved in drug/agent metabolism and several of these metabolites are known to be carcinogens [75]; CYP1A1 mutations may be associated with $\mathrm{CL} / \mathrm{P}$ induced with smoking and detoxification [76]. The role of other downstream targets (NTN1 and $B C L 2$ ) of miR-655-3p in the CL/P etiology remains largely unknown, and mice deficient for either Ntn1 or $B c l 2$ fail to display CL/P and CP.

Our systematic data collection timely summarizes the $\mathrm{CL} / \mathrm{P}$-candidate genes; however, it has some limitations. For example, some genes are from syndromes that include $\mathrm{CL} / \mathrm{P}$ as a feature, but often they do not. These causative genes may be more broadly related to development, but do not contribute to CL/P; CL/P may be secondary to other defects in these syndromes. The current genetic signature of $\mathrm{CL} / \mathrm{P}$ or other complex genetic diseases may be due to the bias of the type of genes that have been studied. Unbiased genome sequencing approaches will likely overcome the limitations and help us discover genes associated with CL/P. Molecular profiling of $\mathrm{CL} / \mathrm{P}$ at the genomic, transcriptomic, regulatory (e.g. enhancer), epigenomic, and proteomic levels in model organisms like mice will provide us with a more detailed and accurate view of how genetic changes cause $\mathrm{CL} / \mathrm{P}$. 


\section{Conclusion}

Our bioinformatics analysis results suggest that a disruption of extracellular cues and their signaling pathways might be a major cause of CL/P, and that miRNAs might play important roles in the CL/P etiology through the regulation of $\mathrm{CL} / \mathrm{P}$ genes. In this study, we found that several miRNAs suppressed cell proliferation in cultured human lip fibroblasts. Among them, we confirmed that miR-655-3p and miR497-5p negatively regulated CL/ P-candidate genes in the cultured cells. This study will have potential relevance to the miRNA-gene pathways and networks, not only in CL/P but also in other organogenesis processes. Therefore, this study will contribute to a better understanding of the mechanisms of CL/ $\mathrm{P}$ and to future clinical interventions to prevent and diagnose $\mathrm{CL} / \mathrm{P}$.

\section{Additional files}

Additional file 1: Table S1. PCR primer sets used in this study. Table S2. Summary of databases searched. Table S3. Genes with significant contribution to human CL/P (identified through single gene studies). Table S4. Genes with significant contribution to human CL/P (identified through multiple genes studies). Table S5. Genes with significant contribution to human CL/P (unknown coding genes). Table S6. Genes without significant contribution to human CL/P. Table S7. CL/P candidate genes with significant signals in GWAS. Table S8. CL/P candidate genes without significant signals in GWAS. Table S9. GO terms enriched with genes associated with cleft lip with/without cleft palate $(\mathrm{CL} / \mathrm{P})$ in humans. Table S10. GO Biological Process terms enriched with human $\mathrm{CL} / \mathrm{P}$ genes (FDR < 0.005). Table S11. GO Molecular Function terms enriched with human $\mathrm{CL} / \mathrm{P}$ genes (FDR < 0.005). Table S12. GO Cellular Component terms enriched with human CL/P genes (FDR < 0.05). Table S13. Top 30 Human Phenotype Ontology Categories. Table S14. KEGG pathways enriched with genes associated with cleft lip with/ without cleft palate (CL/P) in humans. (ZIP $292 \mathrm{~kb}$ )

\section{Abbreviations}

CCDG: Center for Craniofacial and Dental Genetics; CL/P: Cleft lip with or without cleft palate; DAVID: Database for Annotation, Visualization and Integrated Discovery; ECM: Extracellular matrix; FDR: False Discovery Rate; GO: Gene Ontology; GWAS: Genome-wide association study; KEGG: Kyoto Encyclopedia of Genes and Genomes database; MAPK: Mitogen-activated protein kinase; MeSH: Medical Subject Headings; MF: Molecular function; miRNA: microRNA; PI3K: Phosphoinositide 3-kinase; SNP: Single nucleotide polymorphism; TDT: Transmission Disequilibrium Test
\end{abstract}

\section{Acknowledgments}

We thank Mrs. Helena VonVille for her valuable assistance with the systematic review and Dr. Guangchun Han and Dr. Hiroki Yoshioka for assistance with miRNA analysis.

\section{Funding}

This study was supported by grants from the NIH National Institute of Dental and Craniofacial Research (R03DE024759, R03DE026208, R01DE026767, and R03DE026509 to J.l.; R01LM012806, R21CA196508, R03DE027393, and R03DE028103 to Z.Z., and R03DE027711 to P.J.) and a faculty fund from UTHealth School of Dentistry to J.I. The funders had no role in the study design, data collection and analysis, decision to publish, or preparation of the manuscript.

\section{Availability of data and materials}

All the data used in this study are from the public sources cited in our reference list. Additional files, which may be needed to reproduce the results presented in the manuscript, were made available as additional files.

\section{Authors' contributions}

$J$ and $Z Z$ designed the project, MG, ND, GJ, and PJ collected the data, MG, $\mathrm{ND}, \mathrm{AL}, \mathrm{GJ}$, and PJ analyzed the data, AS and MZ carried out experiments, AS and $\boldsymbol{J}$ drafted the manuscript, and $\mathrm{J}$ and $\mathrm{ZZ}$ edited the manuscript. All authors approved the final manuscript.

Ethics approval and consent to participate

Not applicable.

\section{Consent for publication}

Not applicable.

\section{Competing interests}

The authors declare they have no competing interests.

\section{Publisher's Note}

Springer Nature remains neutral with regard to jurisdictional claims in published maps and institutional affiliations.

\section{Author details}

${ }^{1}$ Department of Diagnostic \& Biomedical Sciences, School of Dentistry, The University of Texas Health Science Center at Houston, Houston, TX, USA. ${ }^{2}$ Center for Craniofacial Research, The University of Texas Health Science Center at Houston, Houston, TX, USA. ${ }^{3}$ Center for Precision Health, School of Biomedical Informatics, The University of Texas Health Science Center at Houston, Houston, TX, USA. ${ }^{4}$ Department of Epidemiology, Human Genetics \& Environmental Sciences, School of Public Health, The University of Texas Health Science Center at Houston, Houston, TX, USA. ${ }^{5}$ MD Anderson Cancer Center UTHealth Graduate School of Biomedical Sciences, Houston, TX, USA.

Received: 26 September 2018 Accepted: 16 May 2019

Published online: 23 May 2019

\section{References}

1. Mossey PA, Little J, Munger RG, Dixon MJ, Shaw WC. Cleft lip and palate. Lancet. 2009;374(9703):1773-85.

2. Bender PL. Genetics of cleft lip and palate. J Pediatr Nurs. 2000;15(4):242-9.

3. Bezerra J, Oliveira G, Soares C, Cardoso M, Ururahy M, Neto F, Lima-Neto L, Luchessi A, Silbiger V, Fajardo C. Genetic and non-genetic factors that increase the risk of non-syndromic cleft lip and/or palate development. Oral Dis. 2015;21(3):393-9.

4. Richards L, Plachez C, Ren T. Developmental biology: Frontiers for clinical genetics. Section editors: Roderick R McInnes, e-mail: Jacques Michaud, email:. Mechanisms regulating the development of the corpus callosum and its. Clin Genet. 2004;66(4):276-89.

5. Ghazali N, Rahman NA, Kannan TP, Jaafar S. Screening of transforming growth factor Beta 3 and Jagged2 genes in the Malay population with nonsyndromic cleft lip with or without cleft palate. Cleft Palate Craniofac J. 2015:52(4):e88-94

6. do Rego Borges A, Sá J, Hoshi R, Viena CS, Mariano LC, de Castro Veiga P, Medrado AP, Machado RA, de Aquino SN, Messetti AC. Genetic risk factors for nonsyndromic cleft lip with or without cleft palate in a Brazilian population with high African ancestry. Am J Med Genet A. 2015;167(10): 2344-9.

7. Jia P, Zhao Z. Network.Assisted analysis to prioritize GWAS results: principles, methods and perspectives. Hum Genet. 2014;133(2):125-38.

8. Wang $L$, Jia P, Wolfinger RD, Chen X, Zhao Z. Gene set analysis of genomewide association studies: methodological issues and perspectives. Genomics. 2011;98(1):1-8.

9. Inui M, Martello G, Piccolo S. MicroRNA control of signal transduction. Nat Rev Mol Cell Biol. 2010;11(4):252-63.

10. Ross JS, Carlson JA, Brock G. miRNA: the new gene silencer. Am J Clin Pathol. 2007;128(5):830-6.

11. Lagos-Quintana M, Rauhut $R$, Lendeckel W, Tuschl T. Identification of novel genes coding for small expressed RNAs. Science. 2001;294(5543):853-8. 
12. Sun J, Gong X, Purow B, Zhao Z. Uncovering MicroRNA and transcription factor mediated regulatory networks in glioblastoma. PLoS Comput Biol. 2012;8(7):e1002488.

13. Jiang W, Mitra R, Lin CC, Wang Q, Cheng F, Zhao Z. Systematic dissection of dysregulated transcription factor-miRNA feed-forward loops across tumor types. Brief Bioinform. 2016;17(6):996-1008.

14. Schoen C, Aschrafi A, Thonissen M, Poelmans G, Von den Hoff JW, Carels CEL. MicroRNAs in Palatogenesis and cleft palate. Front Physiol. 2017:8:165

15. Wang Q, Jia P, Cuenco KT, Zeng Z, Feingold E, Marazita ML, Wang L, Zhao Z. Association signals unveiled by a comprehensive gene set enrichment analysis of dental caries genome-wide association studies. PLoS One. 2013; 8(8):e72653.

16. Sangani D, Suzuki A, VonVille H, Hixson JE, Iwata J. Gene mutations associated with temporomandibular joint disorders: a systematic review. OAlib. 2015;2(6)

17. Veronese N, Cereda E, Solmi M, Fowler SA, Manzato E, Maggi S, Manu P, Abe E, Hayashi K, Allard JP, et al. Inverse relationship between body mass index and mortality in older nursing home residents: a meta-analysis of 19,538 elderly subjects. Obes Rev. 2015;16(11):1001-15.

18. Subramanian A, Tamayo P, Mootha VK, Mukherjee S, Ebert BL, Gillette MA, Paulovich A, Pomeroy SL, Golub TR, Lander ES, et al. Gene set enrichment analysis: a knowledge-based approach for interpreting genome-wide expression profiles. Proc Natl Acad Sci U S A. 2005;102(43):15545-50.

19. Chou CH, Chang NW, Shrestha S, Hsu SD, Lin YL, Lee WH, Yang CD, Hong HC, Wei TY, Tu SJ, et al. miRTarBase 2016: updates to the experimentally validated miRNA-target interactions database. Nucleic Acids Res. 2016; 44(D1):D239-47.

20. John B, Enright AJ, Aravin A, Tuschl T, Sander C, Marks DS. Human MicroRNA targets. PLoS Biol. 2004;2(11):e363.

21. Kertesz M, lovino N, Unnerstall U, Gaul U, Segal E. The role of site accessibility in microRNA target recognition. Nat Genet. 2007;39(10):1278-84.

22. Lewis BP, Burge CB, Bartel DP. Conserved seed pairing, often flanked by adenosines, indicates that thousands of human genes are microRNA targets. Cell. 2005;120(1):15-20.

23. Benjamini $Y$, Hochberg $Y$. Controlling the false discovery rate: a practical and powerful approach to multiple testing. J R Stat Soc Ser B Methodol. 1995;57:289-300

24. Suzuki A, Pelikan RC, Iwata J. WNT/beta-catenin signaling regulates multiple steps of Myogenesis by regulating step-specific targets. Mol Cell Biol. 2015; 35(10):1763-76.

25. Suzuki A, Sangani DR, Ansari A, Iwata J. Molecular mechanisms of midfacial developmental defects. Dev Dyn. 2016;245(3):276-93.

26. Obican SG, Finnell RH, Mills JL, Shaw GM, Scialli AR. Folic acid in early pregnancy: a public health success story. FASEB J. 2010;24(11):4167-74

27. Bille C, Winther JF, Bautz A, Murray JC, Olsen J, Christensen K. Cancer risk in persons with oral cleft--a population-based study of 8,093 cases. Am J Epidemiol. 2005;161(11):1047-55.

28. Dunkhase E, Ludwig KU, Knapp M, Skibola CF, Figueiredo JC, Hosking FJ, Ellinghaus $\mathrm{E}$, Landi MT, Ma H, Nakagawa $\mathrm{H}$, et al. Nonsyndromic cleft lip with or without cleft palate and cancer: evaluation of a possible common genetic background through the analysis of GWAS data. Genom Data. 2016; 10:22-9.

29. Taioli E, Ragin C, Robertson L, Linkov F, Thurman NE, Vieira AR. Cleft lip and palate in family members of cancer survivors. Cancer Investig. 2010;28(9): 958-62.

30. Huang JB, Liu YL, Sun PW, Lv XD, Du M, Fan XM. Molecular mechanisms of congenital heart disease. Cardiovasc Pathol. 2010;19(5):e183-93.

31. Torano EG, Garcia MG, Fernandez-Morera JL, Nino-Garcia P, Fernandez AF. The impact of external factors on the epigenome: in utero and over lifetime. Biomed Res Int. 2016;2016:2568635.

32. Kitsiou-Tzeli S, Tzetis M. Maternal epigenetics and fetal and neonatal growth. Curr Opin Endocrinol Diabetes Obes. 2017;24(1):43-6.

33. Wang S, Sun C, Meng Y, Zhang B, Wang X, Su Y, Shi L, Zhao E. A pilot study: screening target miRNAs in tissue of nonsyndromic cleft lip with or without cleft palate. Exp Ther Med. 2017;13(5):2570-6.

34. Ambros $V$. The functions of animal microRNAs. Nature. 2004;431(7006):350-5.

35. Compagnucci C, Fish JL, Schwark M, Tarabykin V, Depew MJ. Pax6 regulates craniofacial form through its control of an essential cephalic ectodermal patterning center. Genesis. 2011;49(4):307-25.

36. Grindley JC, Davidson DR, Hill RE. The role of Pax-6 in eye and nasa development. Development. 1995;121(5):1433-42.
37. Walther C, Gruss P. Pax-6, a murine paired box gene, is expressed in the developing CNS. Development. 1991;113(4):1435-49.

38. Marazita ML, Field LL, Cooper ME, Tobias R, Maher BS, Peanchitlertkajorn S, Liu YE. Nonsyndromic cleft lip with or without cleft palate in China: assessment of candidate regions. Cleft Palate Craniofac J. 2002;39(2):149-56.

39. Marazita ML, Field LL, Cooper ME, Tobias R, Maher BS, Peanchitlertkajorn S, Liu YE. Genome scan for loci involved in cleft lip with or without cleft palate, in Chinese multiplex families. Am J Hum Genet. 2002;71(2):349-64.

40. Muenke M. The pit, the cleft and the web. Nat Genet. 2002;32(2):219-20.

41. Momb J, Lewandowski JP, Bryant JD, Fitch R, Surman DR, Vokes SA, Appling DR. Deletion of Mthfd1I causes embryonic lethality and neural tube and craniofacial defects in mice. Proc Natl Acad Sci U S A. 2013;110(2):549-54.

42. Blanton SH, Henry RR, Yuan Q, Mulliken JB, Stal S, Finnell RH, Hecht JT. Folate pathway and nonsyndromic cleft lip and palate. Birth Defects Res A Clin Mol Teratol. 2011:91(1):50-60.

43. Munger RG, Tamura T, Johnston KE, Feldkamp ML, Pfister R, Cutler R, Murtaugh MA, Carey JC. Oral clefts and maternal biomarkers of folate-dependent onecarbon metabolism in Utah. Birth Defects Res A Clin Mol Teratol. 2011;91(3): 153-61.

44. Boyles AL, Wilcox A, Taylor JA, Shi M, Weinberg CR, Meyer K, Fredriksen A, Ueland PM, Johansen AM, Drevon CA, et al. Oral facial clefts and gene polymorphisms in metabolism of folate/one-carbon and vitamin a: a pathwaywide association study. Genet Epidemiol. 2009;33(3):247-55.

45. Boyles AL, Wilcox AJ, Taylor JA, Meyer K, Fredriksen A, Ueland PM, Drevon CA, Vollset SE, Lie RT. Folate and one-carbon metabolism gene polymorphisms and their associations with oral facial clefts. Am J Med Genet A. 2008;146A(4):440-9.

46. Beaty TH, Murray JC, Marazita ML, Munger RG, Ruczinski I, Hetmanski JB, Liang KY, Wu T, Murray T, Fallin MD, et al. A genome-wide association study of cleft lip with and without cleft palate identifies risk variants near MAFB and ABCA4. Nat Genet. 2010:42(6):525-9.

47. Lee MK, Shaffer JR, Leslie EJ, Orlova E, Carlson JC, Feingold E, Marazita ML, Weinberg SM. Genome-wide association study of facial morphology reveals novel associations with FREM1 and PARK2. PLoS One. 2017;12(4):e0176566.

48. Leslie EJ, Carlson JC, Shaffer JR, Butali A, Buxo CJ, Castilla EE, Christensen K, Deleyiannis FW, Leigh Field L, Hecht JT, et al. Genome-wide meta-analyses of nonsyndromic orofacial clefts identify novel associations between FOXE1 and all orofacial clefts, and TP63 and cleft lip with or without cleft palate. Hum Genet. 2017:136(3):275-86.

49. Leslie EJ, Taub MA, Liu H, Steinberg KM, Koboldt DC, Zhang Q, Carlson JC,

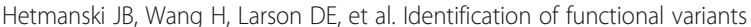
for cleft lip with or without cleft palate in or near PAX7, FGFR2, and NOG by targeted sequencing of GWAS loci. Am J Hum Genet. 2015;96(3):397-411.

50. Butali A, Suzuki S, Cooper ME, Mansilla AM, Cuenco K, Leslie EJ, Suzuki Y, Niimi T, Yamamoto M, Ayanga G, et al. Replication of genome wide association identified candidate genes confirm the role of common and rare variants in PAX7 and VAX1 in the etiology of nonsyndromic $C L(P)$. Am J Med Genet A. 2013;161A(5):965-72.

51. Ma L, Xu M, Li D, Han Y, Wang Z, Yuan H, Ma J, Zhang W, Jiang H, Pan Y, et al. A miRNA-binding-site SNP of MSX1 is associated with NSOC susceptibility. J Dent Res. 2014;93(6):559-64.

52. Li D, Zhang H, Ma L, Han Y, Xu M, Wang Z, Jiang H, Zhang W, Wang L, Pan $Y$. Associations between microRNA binding site SNPs in FGFs and FGFRs and the risk of non-syndromic orofacial cleft. Sci Rep. 2016;6:31054.

53. Li J, Zou J, Li Q, Chen L, Gao Y, Yan H, Zhou B, Li J. Assessment of differentially expressed plasma microRNAs in nonsyndromic cleft palate and nonsyndromic cleft lip with cleft palate. Oncotarget. 2016;7(52):86266-79.

54. Nie X, Wang Q, Jiao K. Dicer activity in neural crest cells is essential for craniofacial organogenesis and pharyngeal arch artery morphogenesis. Mech Dev. 2011;128(3-4):200-7.

55. Huang T, Liu Y, Huang M, Zhao X, Cheng L. Wnt1-cre-mediated conditional loss of dicer results in malformation of the midbrain and cerebellum and failure of neural crest and dopaminergic differentiation in mice. J Mol Cell Biol. 2010;2(3):152-63.

56. Zehir A, Hua LL, Maska EL, Morikawa Y, Cserjesi P. Dicer is required for survival of differentiating neural crest cells. Dev Biol. 2010;340(2):459-67.

57. Sheehy NT, Cordes KR, White MP, Ivey KN, Srivastava D. The neural crest enriched microRNA miR-452 regulates epithelial-mesenchymal signaling in the first pharyngeal arch. Development. 2010;137(24):4307-16.

58. Wu G, Zheng K, Xia S, Wang Y, Meng X, Qin X, Cheng Y. MicroRNA-655-3p functions as a tumor suppressor by regulating ADAM10 and beta-catenin pathway in hepatocellular carcinoma. J Exp Clin Cancer Res. 2016;35(1):89. 
59. Zhao XQ, Liang $B$, Jiang $K$, Zhang HY. Down-regulation of miR-655-3p predicts worse clinical outcome in patients suffering from hepatocellular carcinoma. Eur Rev Med Pharmacol Sci. 2017;21(4):748-52.

60. Chai L, Kang XJ, Sun ZZ, Zeng MF, Yu SR, Ding Y, Liang JQ, Li TT, Zhao J. MiR-497-5p, miR-195-5p and miR-455-3p function as tumor suppressors by targeting hTERT in melanoma A375 cells. Cancer Manag Res. 2018;10:989-1003.

61. Chen Y, Kuang D, Zhao X, Chen D, Wang X, Yang Q, Wan J, Zhu Y, Wang Y, Zhang $S$, et al. miR-497-5p inhibits cell proliferation and invasion by targeting KCa3.1 in angiosarcoma. Oncotarget. 2016;7(36):58148-61.

62. Sun Z, Li A, Yu Z, Li X, Guo X, Chen R. MicroRNA-497-5p Suppresses Tumor Cell Growth of Osteosarcoma by Targeting ADP Ribosylation Factor-Like Protein 2. Cancer Biother Radiopharm. 2017;32(10):371-8.

63. Chen X, Shi C, Wang C, Liu W, Chu Y, Xiang Z, Hu K, Dong P, Han X. The role of miR-497-5p in myofibroblast differentiation of LR-MSCs and pulmonary fibrogenesis. Sci Rep. 2017;7:40958.

64. Wyszynski DF, Beaty TH. Review of the role of potential teratogens in the origin of human nonsyndromic oral clefts. Teratology. 1996;53(5):309-17.

65. Felix TM, Hanshaw BC, Mueller R, Bitoun P, Murray JC. CHD7 gene and nonsyndromic cleft lip and palate. Am J Med Genet A. 2006;140(19):2110-4.

66. Mohamad Shah NS, Salahshourifar I, Sulong S, Wan Sulaiman WA, Halim AS. Discovery of candidate genes for nonsyndromic cleft lip palate through genome-wide linkage analysis of large extended families in the Malay population. BMC Genet. 2016;17:39.

67. Riley BM, Schultz RE, Cooper ME, Goldstein-McHenry T, Daack-Hirsch S, Lee KT, Dragan E, Vieira AR, Lidral AC, Marazita ML, et al. A genome-wide linkage scan for cleft lip and cleft palate identifies a novel locus on 8p11-23. Am J Med Genet A. 2007;143A(8):846-52.

68. Green RM, Feng W, Phang T, Fish JL, Li H, Spritz RA, Marcucio RS, Hooper J, Jamniczky H, Hallgrimsson B, et al. Tfap2a-dependent changes in mouse facial morphology result in clefting that can be ameliorated by a reduction in Fgf8 gene dosage. Dis Model Mech. 2015;8(1):31-43.

69. Milunsky JM, Maher TA, Zhao G, Roberts AE, Stalker HJ, Zori RT, Burch MN, Clemens M, Mulliken JB, Smith R, et al. TFAP2A mutations result in branchiooculo-facial syndrome. Am J Hum Genet. 2008:82(5):1171-7.

70. Xu H, Niu Y, Wang T, Liu S, Xu H, Wang S, Liu J, Ye Z. Novel FGFR1 and KISS1R mutations in Chinese Kallmann syndrome males with cleft lip/palate. Biomed Res Int. 2015;2015:649698.

71. Simonis N, Migeotte I, Lambert N, Perazzolo C, de Silva DC, Dimitrov B, Heinrichs C, Janssens S, Kerr B, Mortier G, et al. FGFR1 mutations cause Hartsfield syndrome, the unique association of holoprosencephaly and ectrodactyly. J Med Genet. 2013;50(9):585-92.

72. Mao XY, Tang SJ. Effects of phenytoin on Satb2 and Hoxa2 gene expressions in mouse embryonic craniofacial tissue. Biochem Cell Biol. 2010; 88(4):731-5

73. Quaderi NA, Schweiger S, Gaudenz K, Franco B, Rugarli El, Berger W, Feldman GJ, Volta M, Andolfi G, Gilgenkrantz S, et al. Opitz G/BBB syndrome, a defect of midline development, is due to mutations in a new RING finger gene on Xp22. Nat Genet. 1997;17(3):285-91.

74. Hsieh EW, Vargervik K, Slavotinek AM. Clinical and molecular studies of patients with characteristics of Opitz G/BBB syndrome shows a novel MID1 mutation. Am J Med Genet A. 2008;146A(18):2337-45.

75. Mescher M, Haarmann-Stemmann T. Modulation of CYP1A1 metabolism: from adverse health effects to chemoprevention and therapeutic options. Pharmacol Ther. 2018;187:71-87.

76. Shi M, Christensen K, Weinberg CR, Romitti P, Bathum L, Lozada A, Morris RW, Lovett M, Murray JC. Orofacial cleft risk is increased with maternal smoking and specific detoxification-gene variants. Am J Hum Genet. 2007; 80(1):76-90

Ready to submit your research? Choose BMC and benefit from:
- fast, convenient online submission
- thorough peer review by experienced researchers in your field
- rapid publication on acceptance
- support for research data, including large and complex data types
- gold Open Access which fosters wider collaboration and increased citations
- maximum visibility for your research: over 100M website views per year
At BMC, research is always in progress.
Learn more biomedcentral.com/submissions

